TRANSACTIONS OF THE

AMERICAN MATHEMATICAL SOCIETY

Volume 363, Number 11, November 2011, Pages 6085-6109

S 0002-9947(2011)05373-0

Article electronically published on May 25, 2011

\title{
ASYMPTOTIC BEHAVIOR FOR A SEMILINEAR SECOND ORDER EVOLUTION EQUATION
}

\author{
CHUNYOU SUN, LU YANG, AND JINQIAO DUAN
}

\begin{abstract}
This paper is devoted to the qualitative analysis for a second order evolution equation $u_{t t}-\Delta u-\Delta u_{t}-\varepsilon \Delta u_{t t}+f(u)=g(x)(\varepsilon \in[0,1])$ with critical nonlinearity. Some uniformly (w.r.t. $\varepsilon \in[0,1])$ asymptotic regularity about the solutions has been established for both $g(x) \in L^{2}(\Omega)$ and $g(x) \in H^{-1}$, which shows that the solutions are exponentially approaching a more regular fixed subset uniformly (w.r.t. $\varepsilon \in[0,1]$ ). As an application of this regularity result, a family $\left\{\mathcal{E}_{\varepsilon}\right\}_{\varepsilon \in[0,1]}$ of finite dimensional exponential attractors has been constructed. Moreover, to characterize the relation with a strongly damped wave equation $(\varepsilon=0)$, the upper semicontinuity, at $\varepsilon=0$, of the global attractors has been proved.
\end{abstract}

\section{INTRODUCTION}

We study the long-time behavior of the following semilinear evolution equation of second order in time:

$\left(E_{\varepsilon}\right) \quad\left\{\begin{array}{l}u_{t t}-\Delta u-\Delta u_{t}-\varepsilon \Delta u_{t t}+f(u)=g(x) \text { in } \Omega \times[0, \infty), \\ \left(u(x, 0), u_{t}(x, 0)\right)=\left(u_{0}(x), v_{0}(x)\right), \\ \left.u\right|_{\partial \Omega}=0,\end{array}\right.$

where $\Omega \subset \mathbb{R}^{N}(N \geq 3)$ is a bounded domain with smooth boundary $\partial \Omega, \varepsilon \in[0,1]$, and the external forcing $g(x)$ is time-independent.

When $\varepsilon=0,\left(E_{0}\right)$ is the usual strongly damped wave equation, and its asymptotic behavior has been studied extensively in terms of attractors; see [4, 5, 17, 13, 16, 23, 25, 32, 35, 36.

For each fixed $\varepsilon_{0}>0$, equation $\left(E_{\varepsilon_{0}}\right)$ is a special form of the so-called improved Boussinesq equation (see 3 , 19, 20, 31]) with damped term $-\Delta u_{t}$, which was used to describe ion-sound waves in plasma by Makhankov 20, 21 and also known to represent other sorts of 'propagation problems' of, for example, lengthways waves in nonlinear elastic rods and ion-sonic waves of space transformations by a weak nonlinear effect (see [3, 10]).

The main purpose of this paper is, based on the global well-posedness results given in [6] and motivated by the dynamical results in [9, 13, 23, 25, 28, 30, 36, 37,

Received by the editors September 23, 2009 and, in revised form, March 15, 2010.

2010 Mathematics Subject Classification. Primary 35G25, 35B40, 35B41.

Key words and phrases. Evolution equation of second order, strongly damped wave equation, asymptotic regularity, critical exponent, attractors.

This work was supported by the NSFC Grants 10601021 and 10926089, the Fund of Physics \& Mathematics of Lanzhou University Grants LZULL200801 and LZULL200903, and the Fundamental Research Funds for the Central Universities Grant lzujbky-2009-48. 
to give some uniform (w.r.t. the parameter $\varepsilon \in[0,1]$ ) qualitative analysis (or a priori estimates) for the solutions of $\left(E_{\varepsilon}\right)$ and then provide some information about the relation between the solutions of $\left(E_{0}\right)$ and those of $\left(E_{\varepsilon}\right)$.

This paper is organized as follows. In $\S 2$, we introduce basic notation and state our main results. In $\S 3$, we recall some abstract results that we will use later. In $\S 4$, we present several dissipative estimates about the solution of $E_{\varepsilon}$, which hold uniformly with respect to $\varepsilon \in[0,1]$. The main results are proved in $\S 5$ and $\S 6$ for $g(x) \in L^{2}(\Omega)$ and $g(x) \in H^{-1}$, respectively. Moreover, as an application, we construct a finite dimensional exponential attractor and prove upper semicontinuity of the global attractor in $\$ 5.5$.

\section{MAin RESUlts}

Before presenting our main results, we first state the basic mathematical assumptions for considering the long-time behaviors of second order evolution equations and then introduce some notation that we will use throughout this paper:

- $f \in \mathcal{C}^{1}(\mathbb{R})$ with $f(0)=0$ and satisfies the following conditions:

$$
\left|f^{\prime}(s)\right| \leq C\left(1+|s|^{\frac{N+2}{N-2}-1}\right) \quad \text { for all } s \in \mathbb{R},
$$

and

$$
\liminf _{|s| \rightarrow \infty} \frac{f(s)}{s}>-\lambda_{1}
$$

where $\lambda_{1}$ is the first eigenvalue of $-\Delta$ on $H_{0}^{1}(\Omega)$.

- Let $A=-\Delta$ with domain $D(A)=H^{2}(\Omega) \cap H_{0}^{1}(\Omega)$, and consider the family of Hilbert spaces $D\left(A^{s / 2}\right), s \in \mathbb{R}$ with the standard inner products and norms, respectively, $\langle\cdot, \cdot\rangle_{D\left(A^{s / 2}\right)}=\left\langle A^{s / 2} \cdot A^{s / 2} \cdot\right\rangle$ and $\|\cdot\|_{D\left(A^{s / 2}\right)}=\left\|A^{s / 2} \cdot\right\|$. In particular, $\langle\cdot, \cdot\rangle$ and $\|\cdot\|$ mean the $L^{2}(\Omega)$ inner product and norm, respectively. We denote

- $\mathcal{H}^{s}=D\left(A^{\frac{1+s}{2}}\right) \times D\left(A^{\frac{1+s}{2}}\right), s \in[0,1]$ with the usual norm

$$
\|(u, v)\|_{\mathcal{H}^{s}}^{2}=\left\|A^{\frac{1+s}{2}} u\right\|^{2}+\left\|A^{\frac{1+s}{2}} v\right\|^{2} .
$$

In particular, we denote $\mathcal{H}=\mathcal{H}^{0}=H_{0}^{1}(\Omega) \times H_{0}^{1}(\Omega)$ and $\|\cdot\|_{\mathcal{H}}=\|\cdot\|_{\mathcal{H}^{0}}$. - For each $(u, v) \in \mathcal{H}$, we define $\|\cdot\|_{\mathcal{H}_{\varepsilon}^{s}}(\varepsilon, s \in[0,1])$ as

$$
\|(u, v)\|_{\mathcal{H}_{\varepsilon}^{s}}^{2}=\left\|A^{\frac{1+s}{2}} u\right\|^{2}+\left\|A^{\frac{s}{2}} v\right\|^{2}+\varepsilon\left\|A^{\frac{1+s}{2}} v\right\|^{2},
$$

and define $\mathcal{H}_{\varepsilon}^{s}$ as

$$
\mathcal{H}_{\varepsilon}^{s}=c l_{\|\cdot\|_{\mathcal{H}}^{s}}\left(\mathcal{H}^{1}\right)
$$

Then $\left(\mathcal{H}_{\varepsilon}^{s},\|\cdot\|_{\mathcal{H}_{\varepsilon}^{s}}\right)$ is a Banach space for every $\varepsilon, s \in[0,1]$.

- $\xi_{u}(t)=\left(u(t), u_{t}(t)\right)$ for any $t \geq 0$.

For clarity, we would like to separate our results into two parts according to the external forcing $g(x) \in L^{2}(\Omega)$ and $g(x) \in H^{-1}$. For the well-posedness, there is no essential difference between the cases $g(x) \in L^{2}(\Omega)$ and $g(x) \in H^{-1}$ if we work in the weakly energy phase space $\mathcal{H}$. However, for the asymptotic regularity (and so the dynamics), there is a big difference: the stationary solutions of $\left(E_{\varepsilon}\right)$ will belong to $H^{2}(\Omega) \cap H_{0}^{1}(\Omega)$ if $g(x) \in L^{2}(\Omega)$, and so one can expect the global attractor $\mathcal{A}_{\varepsilon}$ will be bounded in $\mathcal{H}^{1}$ for this case, but the stationary solutions of $E_{\varepsilon}$ in general 
will only belong to $H_{0}^{1}(\Omega)$ if $g(x) \in H^{-1}$; consequently the global attractor $\mathcal{A}_{\varepsilon}$ now will only be bounded in $H_{0}^{1}(\Omega) \times H_{0}^{1}(\Omega)$.

Part I: $g(x) \in L^{2}(\Omega)$.

We make the following assumption:

Assumption I. $\Omega \subset \mathbb{R}^{N}(N \geq 3)$ is a bounded domain with smooth boundary, $g \in L^{2}(\Omega)$ and $f$ satisfies (2.1) $-(2.2)$ with $f(0)=0$.

The existence-uniqueness of solutions for $\left(E_{\varepsilon_{0}}\right)$ has been proven in [27, 38, by the Faedo-Galerkin method, and then the long-time behavior of the solution of $\left(E_{\varepsilon_{0}}\right)$ via proving the existence of a global attractor in $\mathcal{H}_{\varepsilon_{0}}^{0}$ under Assumption $I$ has been discussed by Xie and Zhong in [33, 34. Recently, Carvalho and Cholewa 6 presented systematic results including the existence-uniqueness and long-time behavior of $\left(E_{\varepsilon_{0}}\right)$ by using the semigroup approach in $\mathcal{H}_{\varepsilon_{0}}^{0}$ (note that $\left(\mathcal{H}_{\varepsilon_{0}}^{0}\right.$, $\left.\|\cdot\|_{\mathcal{H}_{\varepsilon_{0}}^{0}}\right) \cong\left(\mathcal{H},\|\cdot\|_{\mathcal{H}}\right)$ for each fixed $\left.\varepsilon_{0}>0\right)$. They showed that for each $\varepsilon>0$, the solution of $E_{\varepsilon}$ generates a $C^{0}$ semigroup $\left\{S_{\varepsilon}(t)\right\}_{t \geq 0}$ in $\mathcal{H}_{\varepsilon}^{0}$, and also obtain the asymptotic regularity of attractors for the subcritical case, i.e., require the exponent in (2.1) to be strictly less than $\frac{N+2}{N-2}-1$.

The main result of this part is the following asymptotic regularity.

Theorem 2.1. Under Assumption I, there exist a positive constant $\nu$, a bounded (in $\mathcal{H}^{1}$ ) subset $\mathbb{B} \subset \mathcal{H}^{1}$ and a continuous increasing function $Q(\cdot):[0, \infty) \rightarrow[0, \infty)$ such that, for any bounded (in $\mathcal{H}$ ) subset $B \subset \mathcal{H}$,

$$
\forall \varepsilon \in[0,1], \operatorname{dist}_{\mathcal{H}}\left(S_{\varepsilon}(t) B, \mathbb{B}\right) \leq Q\left(\|B\|_{\mathcal{H}}\right) e^{-\nu t} \quad \text { for all } t \geq 0,
$$

where $\mathbb{B}, \nu$ and $Q(\cdot)$ are all independent of $\varepsilon$, and $\left\{S_{\varepsilon}(t)\right\}_{t \geq 0}$ is the semigroup generated by $E_{\varepsilon}$ in $\mathcal{H}_{\varepsilon}^{0}$.

This result says that asymptotically, for each $\left(E_{\varepsilon}\right)$, the solutions are exponentially approaching a more regular fixed subset $\mathbb{B}$ uniformly (w.r.t. $\varepsilon \in[0,1]$ ). Moreover, it implies the following results:

(1) For each $\varepsilon \in[0,1],\left\{S_{\varepsilon}(t)\right\}_{t \geq 0}$ has a global attractor $\mathcal{A}_{\varepsilon}$ in $\mathcal{H}$, and

$$
\bigcup_{\varepsilon \in[0,1]} \mathcal{A}_{\varepsilon} \subset \operatorname{cl}_{\mathcal{H}^{1}}(\mathbb{B}) .
$$

(2) For the case $g \in L^{\infty}$ (e.g., $\left.g(x) \equiv f(0)\right)$ as considered in Carvalho and Cholewa [6], Theorem [2.1] means that we have proved [6, Lemma 3.4] for the critical nonlinear case. Then applying [6, Lemma 3.5 and Lemma 3.6] (which hold certainly for the critical case), we indeed have shown that 6, Theorem 1.3] holds for the critical case.

(3) Based on Theorem 2.1, applying the abstract result devised in [9, 14, 22, for each $\varepsilon \in[0,1]$ we can prove the existence of a finite dimensional exponential attractor $\mathcal{E}_{\varepsilon}$ in $\mathcal{H}$. Moreover, our attraction is uniform (w.r.t. $\varepsilon \in[0,1]$ ) under the $\mathcal{H}$-norm (not only with the $\mathcal{H}_{\varepsilon}^{0}$-norm); see Lemma 5.10.

(4) Since the global attractor $\mathcal{A}_{\varepsilon} \subset \mathcal{E}_{\varepsilon}$, it also implies that the fractal dimension of the global attractor $\mathcal{A}_{\varepsilon}$ is finite. Moreover, based on Theorem 2.1] we show the upper semicontinuity of $\mathcal{A}_{\varepsilon}$ at $\varepsilon=0$; see Lemma 5.12 .

For the proof of Theorem 2.1, the main difficulty comes from the critical nonlinearity and the uniformness w.r.t. $\varepsilon \in[0,1]$. 
Part II: $g(x) \in H^{-1}$.

To prove some asymptotic regularity for this case is more than a dilemma. When $g(x) \in H^{-1}$, we know that in general the solution of the elliptic equation $-\Delta u+$ $f(u)=g(x) \in H^{-1}$ with $\left.u\right|_{\partial \Omega}=0$ only belongs to $H_{0}^{1}(\Omega)$ when $f(\cdot)$ satisfies (2.1)(2.2). So, in this case, we cannot expect any higher regularity of the attractor (if it exists) than $H_{0}^{1}(\Omega) \times H_{0}^{1}(\Omega)$, and indeed we will get a result different from Theorem 2.1 .

In this part, inspired by more recent results in [11, 12, 30, we show that if we shift the solution $\left(u(t), u_{t}(t)\right)$ of $\left(E_{\varepsilon}\right)$ by a proper (fixed) point $(\phi(x), 0)$, then $\left(u(t), u_{t}(t)\right)-(\phi(x), 0)$ will be bounded in some regular space for $t$ sufficiently large.

For this, besides (2.1)-(2.2), we need to assume additionally that $f(\cdot) \in \mathcal{C}^{2}$ and satisfies

$$
\left|f^{\prime \prime}(s)\right| \leq\left\{\begin{array}{ll}
C\left(1+|s|^{\frac{N+2}{N-2}-2}\right) & N=3,4,5, \\
C & N \geq 6,
\end{array} \text { for all } s \in \mathbb{R},\right.
$$

and

$$
f^{\prime}(s) \geq-l \quad \text { for all } s \in \mathbb{R} .
$$

At the same time, from the estimates and calculations given in $\S \S 3$ and 4 , we observe that we only need to estimate for some fixed $\varepsilon_{0} \in(0,1]$, and for the limit case $(\varepsilon=0)$ we refer the reader to 30 . So, without loss of generality, we fix in this part $\varepsilon \equiv 1$, and take the following notation:

Assumption II. $\Omega \subset \mathbb{R}^{N}(N \geq 3)$ is a bounded domain with smooth boundary, $g(x) \in H^{-1}$ and $f$ satisfies (2.1) -(2.2) with $f(0)=0$ and (2.3) -(2.4); take $\varepsilon \equiv 1$, and denote $S(t)=S_{\varepsilon=1}(t)$ for simplicity.

The main result of this part is the following theorem.

Theorem 2.2. Under Assumption II, for each $0 \leq \alpha<\min \left\{1, \frac{N}{2}-1\right\}$, there exist a subset $\mathcal{B}_{\alpha}$, a positive constant $\mu$ and a monotone increasing function $Q_{\alpha}(\cdot)$ : $[0, \infty) \rightarrow[0, \infty)$ such that, for any bounded set $B \subset \mathcal{H}$,

$$
\operatorname{dist}_{\mathcal{H}}\left(S(t) B, \mathcal{B}_{\alpha}\right) \leq Q_{\alpha}\left(\|B\|_{\mathcal{H}}\right) e^{-\mu t} \quad \text { for all } t \geq 0,
$$

where $\mathcal{B}_{\alpha}$ and $Q_{\alpha}(\cdot)$ may depend on $\alpha$, but $\mu$ is independent of $\alpha$, and where $\mathcal{B}_{\alpha}$ satisfies

$$
\mathcal{B}_{\alpha}=\left\{z \in \mathcal{H}:\|z-(\phi(x), 0)\|_{\mathcal{H}^{\alpha}} \leq \Lambda_{\alpha}<\infty\right\}
$$

for some positive constant $\Lambda_{\alpha} . \phi(x)$ is the unique solution of the following elliptic equation:

$$
\left\{\begin{array}{l}
-\Delta \phi+f(\phi)+\eta_{0} \phi=g(x), \quad \text { in } \Omega, \\
\left.\phi\right|_{\partial \Omega}=0,
\end{array}\right.
$$

where the constant $\eta_{0}>0$ is large enough (will be given precisely in (6.1) -(6.2)).

As an immediate result of Theorem 2.2, we know that $\{S(t)\}_{t \geq 0}$ is asymptotically smooth (see [18]) and then has a global attractor $\mathcal{A}^{\prime}$ in $\mathcal{H}$. Moreover, $\mathcal{A}^{\prime}$ has the decomposition $\mathcal{A}^{\prime}=(\phi(x), 0)+\mathcal{A}^{\prime \prime}$ with $\mathcal{A}^{\prime \prime}$ bounded in $H^{1+\alpha}(\Omega) \times H^{1+\alpha}(\Omega)$ for any $\alpha \in\left[0, \min \left\{1, \frac{N}{2}-1\right\}\right)$. Furthermore, we can show that if the initial data belongs to $(\phi(x), 0)+\mathcal{H}^{\alpha}$, then the corresponding solution $\left(u(t), u_{t}(t)\right)$ will also lie in $(\phi(x), 0)+\mathcal{H}^{\alpha}$ for all $t \geq 0$ and $\left\|\left(u(t)-\phi(x), u_{t}(t)\right)\right\|_{\mathcal{H}^{\alpha}}$ uniformly (w.r.t. time $t$ and initial data) bounded; see Lemma 6.6. 
Remark 2.3. Comparing with Assumption I, Assumption II relaxes the regularity of the forcing term $g(x)$ to $H^{-1}$ (which is the weakest forcing term if we work in the weak energy phase space $\mathcal{H}_{\varepsilon}^{0}$ ), but with the price that we require two additional technical assumptions (2.3)-(2.4). Especially, (2.4) is a restriction and stronger than (2.2) to some extent (although it is reasonable for the critical polynomial nonlinearity case). How to remove such technical assumptions would be interesting. At the same time, we also remark that assumptions (2.1)-(2.2) are sufficient for the existence of a compact global attractor in $\mathcal{H}$ for the case $g(x) \in H^{-1}$ (e.g., see [25, 29, 36]).

Hereafter, we will also use the following notation (see, e.g., [13]): denote by $\mathfrak{J}$ the space of continuous increasing functions $J: \mathbb{R}^{+} \rightarrow \mathbb{R}^{+}$, and by $\mathfrak{D}$ the space of continuous decreasing functions $\beta: \mathbb{R}^{+} \rightarrow \mathbb{R}^{+}$such that $\beta(\infty)<1$. Moreover, $C, C_{i}$ are the generic constants, and $Q(\cdot), Q_{i}(\cdot) \in \mathfrak{J}$ are generic functions, which are all independent of $\varepsilon$; otherwise we will point out clearly. We also denote $\|A\|_{X}=$ $\sup _{x \in X}\|x\|_{X}$ for any $A \subset\left(X,\|\cdot\|_{X}\right)$.

\section{Preliminaries}

In this section, we recall some results used in the main part of the paper.

The first result comes from [13, which will be used to prove the asymptotic regularity for the case $g \in L^{2}(\Omega)$.

Lemma 3.1 ([13]). Let $X, V$ be two Banach spaces and $\{T(t)\}_{t \geq 0}$ be a $C^{0}$-semigroup on $X$ with a bounded absorbing set $\bar{B} \subset X$. For every $x \in \bar{B}$, assume that there exist two solution operators $V_{x}(t)$ on $X$ and $U_{x}(t)$ on $V$ satisfying the following properties:

i) For any two vectors $y \in X$ and $z \in V$ satisfying $y+z=x$,

$$
T(t) x=V_{x}(t) y+U_{x}(t) z \text { for any } t \geq 0 .
$$

ii) There exists $\alpha \in \mathfrak{D}$ such that

$$
\sup _{x \in \bar{B}}\left\|V_{x}(t) y\right\|_{X} \leq \alpha(t)\|y\|_{X}, \quad \forall y \in \bar{B} .
$$

iii) There are $\beta \in \mathfrak{D}$ and $J \in \mathfrak{J}$ such that

$$
\sup _{x \in \bar{B}}\left\|U_{x}(t) z\right\|_{V} \leq \beta(t)\|z\|_{V}+J(t), \quad \forall z \in V .
$$

Then, there exist positive constants $\rho, K, \omega$ such that

$$
\operatorname{dist}_{X}\left(T(t) \bar{B}, B_{V}(\rho)\right) \leq K e^{-\omega t} \text { for all } t \geq 0,
$$

where $B_{V}(\rho)=\left\{z \in V:\|z\|_{V} \leq \rho\right\}$.

Next we recall a criterion for the upper semicontinuity of attractors.

Lemma $3.2\left([18,26)\right.$. Let $\left\{T_{\lambda}(t)\right\}_{t \geq 0}(\lambda \in \Lambda)$ be a family of semigroups defined on the Banach space $X$, and for each $\lambda \in \Lambda$, let $\left\{T_{\lambda}(t)\right\}_{t \geq 0}$ have a global attractor $\mathcal{A}_{\lambda}$. Assume further that $\lambda_{0}$ is a nonisolated point of $\Lambda$ and that there exist $s>0$, $t_{0}>0$ and a compact set $K \subset X$ such that

$$
\begin{aligned}
& \bigcup_{\lambda \in \mathcal{N}_{\Lambda}\left(\lambda_{0}, s\right)} \mathcal{A}_{\lambda} \subset K, \text { and } \\
& \text { if } \lambda_{n} \rightarrow \lambda_{0} \text { and } x_{n} \rightarrow x_{0}\left(x_{n} \in \mathcal{A}_{\lambda_{n}} \text { as } n \neq 0\right) \text {, then } T_{\lambda_{n}}\left(t_{0}\right) x_{n} \rightarrow T_{\lambda_{0}}\left(t_{0}\right) x_{0} .
\end{aligned}
$$


Then the global attractors $\mathcal{A}_{\lambda}$ are upper semicontinuous on $\Lambda$ at $\lambda=\lambda_{0}$; that is,

$$
\lim _{\Lambda \ni \lambda \rightarrow \lambda_{0}} \operatorname{dist}_{X}\left(\mathcal{A}_{\lambda}, \mathcal{A}_{\lambda_{0}}\right)=0 .
$$

We also recall a Gronwall-type inequality; for the proof, please see [17.

Lemma 3.3. Let $\Lambda: \mathbb{R}^{+} \rightarrow \mathbb{R}^{+}$be an absolutely continuous function satisfying

$$
\frac{d}{d t} \Lambda(t)+2 \eta \Lambda(t) \leq h(t) \Lambda(t)+k,
$$

where $\eta>0, k \geq 0$ and $\int_{s}^{t} h(\tau) d \tau<\eta(t-s)+m$ for all $t \geq s \geq 0$ and some $m \geq 0$. Then,

$$
\Lambda(t) \leq \Lambda(0) e^{m} e^{-\eta t}+\frac{k e^{m}}{\eta}, \quad \forall t \geq 0 .
$$

\section{UNiformLy DECAYing ESTIMATES in $\mathcal{H}$}

In this section, we always assume that only (2.1)-(2.2) hold, and $g(x)$ only belongs to $H^{-1}$ (so all results obtained in this section certainly hold for the case $\left.g(x) \in L^{2}(\Omega)\right)$.

The main purpose of this section is to deduce some dissipative estimates about the semigroups $\left\{S_{\varepsilon}(t)\right\}_{t \geq 0}(\varepsilon \in[0,1])$ in $\mathcal{H}$.

The existence of a bounded absorbing set for $\left\{S_{0}(t)\right\}_{t \geq 0}$ in $H_{0}^{1}(\Omega) \times L^{2}(\Omega)$ was established in many references under the assumptions (2.1)-(2.2); e.g., see [2, 5, 23. Recently, Pata and Zelik 25] showed further that indeed there is a bounded absorbing set for $\left\{S_{0}(t)\right\}_{t \geq 0}$ in $\mathcal{H}$, and the authors in [6, 33] obtained the existence of a bounded absorbing set for each fixed $\varepsilon \in(0,1]$. Here, using the method in 23, 25 for a strongly damped wave equation, we will show that the radius of the absorbing set of $\left\{S_{\varepsilon}(t)\right\}_{t \geq 0}$ in $\mathcal{H}$ can be chosen to be independent of $\varepsilon \in[0,1]$.

Lemma 4.1. There exists a positive constant $M$, which depends only on $|\Omega|$, $\|g\|_{H^{-1}}$ and the coefficients of (2.1)-(2.2), satisfying that for any $\varepsilon \in[0,1]$ and any bounded (in $\mathcal{H}_{\varepsilon}^{0}$ ) subset $B \subset \mathcal{H}_{\varepsilon}^{0}$, there is a $t_{B}=t\left(\|B\|_{\mathcal{H}_{\varepsilon}^{0}}\right)>0$ (which depends only on the bound of $\|B\|_{\mathcal{H}_{\varepsilon}^{0}}$ ) such that

$$
\left\|S_{\varepsilon}(t) z\right\|_{\mathcal{H}}^{2} \leq M \quad \text { for all } t \geq t_{B} \text { and all } z \in B,
$$

where both $t_{B}$ and $M$ are independent of $\varepsilon \in[0,1]$.

Proof. Throughout the proof, the generic constants $C, C_{j}(j=1,2, \cdots)$ are independent of $\varepsilon$, and $\Pi_{i}$ denotes the projector from $X_{1} \times X_{2}$ to $X_{i}, i=1,2$.

For clarity, we separate the proof into three claims.

Claim 1. There exists an $M_{1}$ (independent of $B$ and $\varepsilon$ ) such that, $\forall \varepsilon \in[0,1]$,

$$
\left\|S_{\varepsilon}(t) B\right\|_{\mathcal{H}_{\varepsilon}^{0}}^{2}=\left\|S_{\varepsilon}(t) B\right\|_{H_{0}^{1}(\Omega) \times L^{2}(\Omega)}^{2}+\varepsilon\left\|\Pi_{2} S_{\varepsilon}(t) B\right\|_{H_{0}^{1}(\Omega)}^{2} \leq M_{1} \quad \text { as } t \geq T_{1 B},
$$

where $T_{1 B}=T_{1}\left(\|B\|_{\mathcal{H}_{\varepsilon}^{0}}\right)$ depends on $\|B\|_{\mathcal{H}_{\varepsilon}^{0}}$ but not on $\varepsilon$.

Multiplying $E_{\varepsilon}$ by $u_{t}+\theta u$ (here and after, note that the multiplication holds in a Faedo-Galerkin scheme; however, due to the global well-posed result given in [6], the estimates hold in the limit) with $\theta \ll 1$, which will be determined later, we 
obtain that

$$
\frac{d}{d t} E_{1 u}(t)+2 G_{1 u}(t) \leq 2\left\|\nabla u_{t}\right\|\|g\|_{H^{-1}}+2 \theta\|\nabla u\|\|g\|_{H^{-1}}
$$

where

$$
\begin{gathered}
E_{1 u}(t)=\left\|u_{t}(t)+\theta u(t)\right\|^{2}+\varepsilon\left\|\nabla\left(u_{t}(t)+\theta u(t)\right)\right\|^{2}+\left(1+\theta-\varepsilon \theta^{2}\right)\|\nabla u(t)\|^{2} \\
-\theta^{2}\|u(t)\|^{2}+2 \int_{\Omega} F(u(t)) d x \\
G_{1 u}(t)=(1-\varepsilon \theta)\left\|\nabla u_{t}(t)\right\|^{2}-\theta\left\|u_{t}(t)\right\|^{2}+\theta\|\nabla u(t)\|^{2}+\theta \int_{\Omega} f(u(t)) u(t) d x
\end{gathered}
$$

and

$$
F(u)=\int_{0}^{u(x, t)} f(s) d s .
$$

Then, from assumptions (2.1)-(2.2) and using Poincaré's inequality, we have

$$
E_{1 u}(t) \leq C_{1}\left(\left\|u_{t}\right\|^{2}+\varepsilon\left\|\nabla u_{t}\right\|^{2}\right)+C_{2}\left(1+\|\nabla u\|^{\frac{2 N}{N-2}}\right)
$$

and

$$
F(s) \geq-\frac{\lambda}{2} s^{2}-c_{1} \quad \text { for all } s \in \mathbb{R} \text { with some } \lambda \in\left(0, \lambda_{1}\right) .
$$

Noting that $\|x+y\|^{2} \geq \frac{1}{2}\|x\|^{2}-\|y\|^{2}$ holds for any $x, y \in L^{2}(\Omega)$, we have

$$
E_{1 u}(t) \geq \frac{1}{2}\left(\left\|u_{t}\right\|^{2}+\varepsilon\left\|\nabla u_{t}\right\|^{2}\right)+\left(1+\theta-\theta^{2}-\varepsilon \theta^{2}-\frac{2 \theta^{2}+\lambda}{\lambda_{1}}\right)\|\nabla u\|^{2}-2 c_{1}|\Omega|,
$$

where the positive constant $c_{1}$ depends only on $f(\cdot)$ (from (4.4)).

For $G_{1 u}(t)$, we have

$$
G_{1 u}(t) \geq\left(1-\theta-\frac{\theta}{\lambda_{1}}\right)\left\|\nabla u_{t}\right\|^{2}+\theta\left(1-\frac{\lambda}{\lambda_{1}}\right)\|\nabla u\|^{2}-\theta c_{2}|\Omega|,
$$

where $\lambda \in\left(0, \lambda_{1}\right)$ comes from (2.2) and the constant $c_{2}$ depends only on $f(\cdot)$.

At the same time, by the Cauchy-Schwarz inequality, we have

$2\left\|\nabla u_{t}\right\|\|g\|_{H^{-1}}+2 \theta\|\nabla u\|\|g\|_{H^{-1}} \leq\left(1-\theta-\frac{\theta}{\lambda_{1}}\right)\left\|\nabla u_{t}\right\|^{2}+\theta\left(1-\frac{\lambda}{\lambda_{1}}\right)\|\nabla u\|^{2}+C_{\theta}\|g\|_{H^{-1}}^{2}$.

Substituting (4.6) and (4.7) into (4.2), we obtain

$$
\frac{d}{d t} E_{1 u}(t)+\left(1-\theta-\frac{\theta}{\lambda_{1}}\right)\left\|\nabla u_{t}\right\|^{2}+\theta\left(1-\frac{\lambda}{\lambda_{1}}\right)\|\nabla u\|^{2} \leq C_{\theta}\|g\|_{H^{-1}}^{2}+2 \theta c_{2}|\Omega| .
$$

Hence, we first take $\theta$ small enough such that

$$
1+\theta-2 \theta^{2}-\frac{2 \theta^{2}+\lambda}{\lambda_{1}}>0 \text { and } 1-\theta-\frac{\theta}{\lambda_{1}}>0,
$$

and then applying the Gronwall-type inequality, [23, Lemma 1], to (4.8), and combining with (4.3) and (4.5), we have

$$
E_{1 u}(t) \leq \sup _{\varepsilon \in[0,1]} \sup _{z \in \mathcal{H}_{\varepsilon}^{0}}\left\{E_{\varepsilon}(z): \delta\|z\|_{\mathcal{H}_{\varepsilon}^{0}}^{2} \leq 2 C_{\theta}\|g\|_{H^{-1}}^{2}+4 c_{2}|\Omega|\right\} \text { for all }\left(u_{0}, v_{0}\right) \in B
$$


provided that

$$
t \geq T_{1 B}=\frac{2 c_{1}|\Omega|+C_{1}\|B\|_{\mathcal{H}_{\varepsilon}^{0}}+C_{2}\left(1+\|B\|_{\mathcal{H}_{\varepsilon}^{0}}^{\frac{2 N}{N-2}}\right)}{C_{\theta}\|g\|_{H^{-1}}^{2}+2 c_{2}|\Omega|},
$$

where $\delta=\min \left\{\left(1-\theta-\frac{\theta}{\lambda_{1}}\right), \theta\left(1-\frac{\lambda}{\lambda_{1}}\right)\right\}$ and (from (4.3) $)$

$$
E_{\varepsilon}(z):=C_{1}\|z\|_{\mathcal{H}_{\varepsilon}^{0}}^{2}+C_{2}\left(1+\|z\|_{\mathcal{H}_{\varepsilon}^{0}}^{2}\right)^{\frac{N}{N-2}}
$$

for any $z \in \mathcal{H}_{\varepsilon}^{0}$.

Then, noticing (4.5) and (4.9), Claim 1 follows from (4.10) immediately.

Claim 2. There exists an $M_{2}$ (independent of $B$ and $\varepsilon$ ) such that

$$
\forall \varepsilon \in[0,1], \int_{T_{1 B}}^{\infty}\left\|\Pi_{2} S_{\varepsilon}(s) B\right\|_{H_{0}^{1}(\Omega)}^{2} d s \leq M_{2},
$$

where $T_{1 B}$ is given in Claim 1 ,

Multiplying $\left(E_{\varepsilon}\right)$ by $u_{t}$, we have

$\frac{1}{2} \frac{d}{d t}\left(\left\|u_{t}\right\|^{2}+\|\nabla u\|^{2}+\varepsilon\left\|\nabla u_{t}\right\|^{2}+2 \int_{\Omega} F(u) d x-2\langle g(x), u\rangle_{H_{0}^{1}(\Omega), H^{-1}}\right)+\left\|\nabla u_{t}\right\|^{2} \leq 0$.

Then, for any $t \geq T_{1 B}$, integrating (4.12) over $\left[T_{1 B}, t\right]$ and using Claim 1 we have

$$
\int_{T_{1 B}}^{t}\left\|\Pi_{2} S_{\varepsilon}(s) B\right\|_{H_{0}^{1}(\Omega)}^{2} d s \leq 2 M_{1}+2\|g\|_{H^{-1}}^{2}+2 C^{\prime}\left(|\Omega|+M_{1}^{\frac{N}{N-2}}\right),
$$

where the constant $C^{\prime}$ depends only on the constant $C$ in (2.1).

Claim 3. There exists an $M_{3}$ (independent of $B$ and $\varepsilon$ ) such that

$$
\forall \varepsilon \in[0,1],\left\|\Pi_{2} S_{\varepsilon}(t) B\right\|_{H_{0}^{1}(\Omega)}^{2} \leq M_{3} \text { as } t \geq T_{1 B}+1 .
$$

Similar to the proof of [25, Lemma 3.5], multiplying $\left(E_{\varepsilon}\right)$ by $u_{t t}$, we have

$$
\frac{d}{d t} E_{2 u}(t)+2\left\|u_{t t}\right\|^{2}+2 \varepsilon\left\|\nabla u_{t t}\right\|^{2}=2\left\|\nabla u_{t}\right\|^{2}+2\left\langle f^{\prime}(u) u_{t}, u_{t}\right\rangle,
$$

where $\langle\cdot, \cdot\rangle$ is the $L^{2}$-inner product and

$$
E_{2 u}(t)=\left\|\nabla u_{t}(t)\right\|^{2}+2\left\langle\nabla u, \nabla u_{t}\right\rangle+2\left\langle f(u), u_{t}\right\rangle-2\left\langle g(x), u_{t}\right\rangle_{H_{0}^{1}(\Omega), H^{-1}} .
$$

Then, as $t \geq T_{1 B}$, using Claim 1 and (2.1), we have

$$
\frac{d}{d t} E_{2 u}(t)+2\left\|u_{t t}\right\|^{2}+2 \varepsilon\left\|\nabla u_{t t}\right\|^{2} \leq C\left(1+M_{1}^{\frac{N}{N-2}}\right)\left\|\nabla u_{t}\right\|^{2}
$$

and

(4.16)

$\frac{1}{2}\left\|\nabla u_{t}\right\|^{2}-C_{1}\left(1+M_{1}^{\frac{N}{N-2}}+\|g\|_{H^{-1}}^{2}\right) \leq E_{2 u}(t) \leq 2\left\|\nabla u_{t}\right\|^{2}+C_{2}\left(1+M_{1}^{\frac{N}{N-2}}+\|g\|_{H^{-1}}^{2}\right)$.

On the other hand, from Claim 2 we know that for each $\left(u_{0}, v_{0}\right) \in B$, there is a time $t_{0} \in\left[T_{1 B}, T_{1 B}+1\right]$ such that

$$
\left\|\Pi_{2} S_{\varepsilon}\left(t_{0}\right)\left(u_{0}, v_{0}\right)\right\|_{H_{0}^{1}(\Omega)}^{2} \leq M_{2},
$$

where $t_{0}$ depends on $\left(u_{0}, v_{0}\right)$. 
Therefore, as $t \geq T_{1 B}+1$, for each $\left(u_{0}, v_{0}\right) \in B$, integrating (4.15) over $\left[t_{0}, t\right]$ and applying (4.16)-(4.17), we obtain that

$$
\begin{aligned}
& \frac{1}{2}\left\|\nabla u_{t}(t)\right\|^{2}+2 \int_{T_{1 B}+1}^{t}\left(\left\|u_{t t}(s)\right\|^{2}+\varepsilon\left\|\nabla u_{t t}(s)\right\|^{2}\right) d s \\
& \leq 2 M_{2}+\left(C_{1}+C_{2}\right)\left(1+M_{1}^{\frac{N}{N-2}}+\|g\|_{H^{-1}}^{2}\right)+C\left(1+M_{1}^{\frac{N}{N-2}}\right) M_{2}
\end{aligned}
$$

this show that Claim 3 holds.

Now, we can complete our proof by taking

$$
M=M_{1}+M_{3} \text { and } t_{B}=T_{1 B}+1 .
$$

Remark 4.2. Observe that from (4.1), (4.8), (4.3) and (4.5) we can also deduce that, for any $\varepsilon \in[0,1]$ and any $B \subset \mathcal{H}_{\varepsilon}^{0}$,

$$
\left\|S_{\varepsilon}(t) B\right\|_{\mathcal{H}_{\varepsilon}^{0}}^{2} \leq Q\left(\|B\|_{\mathcal{H}_{\varepsilon}^{0}}\right) \text { for all } t \geq 0,
$$

where $Q(\cdot) \in \mathfrak{J}$ is independent of $B$ and $\varepsilon$.

Moreover, if $B$ is bounded in $\mathcal{H}$, then we can obtain

$$
\forall \varepsilon \in[0,1], t \geq 0,\left\|S_{\varepsilon}(t) B\right\|_{\mathcal{H}}^{2} \leq C_{\|B\|_{\mathcal{H}}}
$$

for some constant $C_{\|B\|_{\mathcal{H}}}$ which depends only on $\|B\|_{\mathcal{H}}$. Indeed, from the fact that there is a constant $c_{1}$ such that $c_{1}\|\cdot\|_{\mathcal{H}} \geq\|\cdot\|_{\mathcal{H}_{\varepsilon}^{0}}$ for any $\varepsilon \in[0,1]$, (4.19) can be obtained just by repeating the proof of Lemma 4.1 and taking $t_{0}=0$ in (4.17) since $B$ is bounded in $\mathcal{H}$.

On the other hand, from the proof of Claim 3 above, we can get further estimates about $u_{t t}$ :

$$
\forall \varepsilon \in[0,1], \int_{T_{1 B}+1}^{\infty}\left(\left\|u_{t t}(s)\right\|^{2}+\varepsilon\left\|\nabla u_{t t}(s)\right\|^{2}\right) d s \leq M_{3} \quad \text { for all }\left(u_{0}, v_{0}\right) \in B .
$$

Then, similar to [25, Lemma 3.6], we indeed can deduce the following estimates:

Lemma 4.3. There exists an $M_{4}$ such that for any $\varepsilon \in[0,1]$ and any bounded (in $\left.\mathcal{H}_{\varepsilon}^{0}\right)$ subset $B \subset \mathcal{H}_{\varepsilon}^{0}$,

$$
\left\|u_{t t}(t)\right\|^{2}+\varepsilon\left\|\nabla u_{t t}(t)\right\|^{2}+\int_{T_{1 B}+2}^{t}\left\|\nabla u_{t t}(s)\right\|^{2} d s \leq M_{4} \quad \text { for all } t \geq T_{1 B}+2,
$$

where $\left(u(t), u_{t}(t)\right)=S_{\varepsilon}(t)\left(u_{0}, v_{0}\right)\left(\left(u_{0}, v_{0}\right) \in B\right), T_{1 B}$ is the time given in Claim 1, and $M_{4}$ is independent of $B$ and $\varepsilon$.

For later applications, we present some Hölder continuity of $\left\{S_{\varepsilon}(t)\right\}_{t \geq 0}$ in $\mathcal{H}_{\varepsilon}^{0}$, which has been obtained in [6] for each fixed $\varepsilon \in(0,1]$ and [23] for $\varepsilon=0$.

Lemma 4.4. For any $\varepsilon \in[0,1]$ and any bounded (in $\mathcal{H}_{\varepsilon}^{0}$ ) subset $B \subset \mathcal{H}_{\varepsilon}^{0}$, there is a constant $C_{\|B\|_{\mathcal{H}_{\varepsilon}^{0}}}$ which depends only on $\|B\|_{\mathcal{H}_{\varepsilon}^{0}}$ such that

$$
\left\|S_{\varepsilon}(t) z_{1}-S_{\varepsilon}(t) z_{2}\right\|_{\mathcal{H}_{\varepsilon}^{0}} \leq e^{C_{\|B\|_{\mathcal{H}_{\varepsilon}^{0}}} t}\left\|z_{1}-z_{2}\right\|_{\mathcal{H}_{\varepsilon}^{0}}, \forall t \geq 0, z_{i} \in B
$$

and

$$
\left\|S_{\varepsilon}(t) z_{1}-S_{\varepsilon}(t) z_{2}\right\|_{\mathcal{H}} \leq e^{C_{\|B\|_{\mathcal{H}_{\varepsilon}^{0}}} t}\left\|z_{1}-z_{2}\right\|_{\mathcal{H}_{\varepsilon}^{0}}^{\frac{1}{2}}, \forall t \geq T_{1 B}+2, z_{i} \in B
$$


Proof. Let $\left(u^{i}(t), u_{t}^{i}(t)\right)$ be the solution of $\left(E_{\varepsilon}\right)$ corresponding to the initial data $z_{i} \in B$. Then the difference $\bar{u}=u^{1}-u^{2}$ satisfies

$$
\bar{u}_{t t}-\Delta \bar{u}_{t}-\Delta \bar{u}-\varepsilon \Delta \bar{u}_{t t}+f\left(u^{1}\right)-f\left(u^{2}\right)=0
$$

with initial data $\left(\bar{u}(0), \bar{u}_{t}(0)\right)=z_{1}-z_{2}$.

Then, as that in 23, we can obtain (4.21) through multiplying (4.23) by $\bar{u}_{t}$ (where we need to use (4.18)).

For (4.22), when $t \geq T_{1 B}+2$, we have

$$
\left\|\nabla \bar{u}_{t}(t)\right\|^{2} \leq\left\|\bar{u}_{t t}\right\|\left\|\bar{u}_{t}\right\|+\|\nabla \bar{u}\|\left\|\nabla \bar{u}_{t}\right\|+\varepsilon\left\|\nabla \bar{u}_{t t}\right\|\left\|\nabla \bar{u}_{t}\right\|+C_{M}\|\nabla \bar{u}\|\left\|\nabla \bar{u}_{t}\right\|,
$$

and then, combining with Lemma 4.3 and (4.21), we have

$$
\begin{aligned}
\frac{1}{4}\left\|\nabla \bar{u}_{t}(t)\right\|^{2} & \leq \sqrt{M_{4}}\left(\left\|\bar{u}_{t}\right\|+\sqrt{\varepsilon}\left\|\nabla \bar{u}_{t}\right\|\right)+C_{M}\|\nabla \bar{u}\|^{2} \\
& \leq \sqrt{M_{4}} e^{C_{\|B\|_{\mathcal{H}_{\varepsilon}^{0}}} t}\left\|z_{1}-z_{2}\right\|_{\mathcal{H}_{\varepsilon}^{0}}+C_{M} e^{2 C_{\|B\|_{\mathcal{H}_{\varepsilon}^{0}}} t}\left\|z_{1}-z_{2}\right\|_{\mathcal{H}_{\varepsilon}^{0}}^{2} \\
& \leq C_{M, M_{4}} e^{C_{M} t}\left\|z_{1}-z_{2}\right\|_{\mathcal{H}_{\varepsilon}^{0}} \text { as } t \geq T_{1 B}+2,
\end{aligned}
$$

which, combining with (4.21) again, implies (4.22) immediately.

Hereafter, we denote the uniformly (w.r.t. $\varepsilon \in[0,1]$ ) bounded absorbing set obtained in Lemma 4.1 as $B_{0}$, i.e.,

$$
B_{0}=\left\{z \in \mathcal{H}:\|z\|_{\mathcal{H}}^{2} \leq M\right\},
$$

and denote by $\Lambda_{0}$ the time such that Lemma 4.1 and Lemma 4.3 hold for $B_{0}$; i.e.,

$$
\left\|S_{\varepsilon}(t) B_{0}\right\|_{\mathcal{H}}^{2}+\left\|u_{t t}(t)\right\|^{2}+\varepsilon\left\|\nabla u_{t t}(t)\right\|^{2}+\int_{T_{1 B}+2}^{t}\left\|\nabla u_{t t}(s)\right\|^{2} d s \leq \bar{M}=M+M_{4}
$$

holds for any $\varepsilon \in[0,1]$ and all $t \geq \Lambda_{0}$. Moreover, similar to Remark 4.2 noting now that $B_{0}$ is bounded in $\mathcal{H}$, we have

$$
\forall \varepsilon \in[0,1],\left\|S_{\varepsilon}(t) B_{0}\right\|_{\mathcal{H}}^{2} \leq C_{M} \text { for all } t \geq 0 .
$$

5. PART I: $g(x) \in L^{2}(\Omega)$

Throughout this section, we always (only) assume that Assumption I holds.

5.1. Decomposition of the equation. For the nonlinear function $f$ satisfying (2.1)-(2.2), from [1] (see also [13, 23, 28, 35] for our situation) we know that $f$ allows the following decomposition $f=f_{0}+f_{1}$, where $f_{0}, f_{1} \in \mathcal{C}^{1}(\mathbb{R})$ and satisfy

$$
\begin{gathered}
\left|f_{0}(u)\right| \leq C|u|^{\frac{N+2}{N-2}} \quad \text { for all } u \in \mathbb{R}, \\
f_{0}(u) u \geq 0 \quad \text { for all } u \in \mathbb{R}, \\
\left|f_{1}(u)\right| \leq C(1+|u|) \quad \text { for all } u \in \mathbb{R}, \\
\liminf _{|u| \rightarrow \infty} \frac{f_{1}(u)}{u}>-\lambda_{1} .
\end{gathered}
$$

For example, from (2.2) we know that there are $s_{1} \geq 0$ and $\lambda<\lambda_{1}$ such that

$$
f(u) u \geq-\lambda u^{2} \quad \text { as }|u| \geq s_{1},
$$

and from (2.1) we know that there is a constant $C_{1}>0$ such that

$$
|f(u)| \leq C_{1}\left(1+|u|^{\frac{N+2}{N-2}}\right) \leq 2 C_{1}|u|^{\frac{N+2}{N-2}} \quad \text { as }|u| \geq 1 .
$$


Take the cutoff function $\varphi(\cdot):[0, \infty) \rightarrow[0,1]$ as follows:

$$
\varphi(s)= \begin{cases}1 & \text { as } s \geq s_{1}+2 \\ 0 & \text { as } s \leq s_{1}+1\end{cases}
$$

Then, we can take $f_{0}(s)=\varphi(|s|)(f(s)+\lambda s)$ and $f_{1}(s)=f(s)-f_{0}(s), \forall s \in \mathbb{R}$.

We will follow the idea (method) in 23, 28, 35, 37, to deduce the asymptotic regularity. Decomposing the solution $S_{\varepsilon}(t)\left(u_{0}, v_{0}\right)=\left(u(t), u_{t}(t)\right)$ into the sum

$$
S_{\varepsilon}(t)\left(u_{0}, v_{0}\right)=D_{\varepsilon}(t)\left(u_{0}, v_{0}\right)+K_{\varepsilon}(t)\left(u_{0}, v_{0}\right)
$$

for any $t \geq 0$ and any $\left(u_{0}, v_{0}\right) \in \mathcal{H}$, where $D_{\varepsilon}(t)\left(u_{0}, v_{0}\right)=\left(v(t), v_{t}(t)\right)$ and $K_{\varepsilon}(t)\left(u_{0}, v_{0}\right)=\left(w(t), w_{t}(t)\right)$ are the solution of the following equations:

$$
\left\{\begin{array}{l}
v_{t t}-\Delta v_{t}-\Delta v-\varepsilon \Delta v_{t t}+f_{0}(v)=0 \text { in } \Omega \times[0, \infty), \\
\left(v(0), v_{t}(0)\right)=\left(u_{0}, v_{0}\right),\left.v\right|_{\partial \Omega}=0,
\end{array}\right.
$$

and

$$
\left\{\begin{array}{l}
w_{t t}-\Delta w_{t}-\Delta w-\varepsilon \Delta w_{t t}+f(u)-f_{0}(v)=g(x) \text { in } \Omega \times[0, \infty), \\
\left(w(0), w_{t}(0)\right)=(0,0),\left.w\right|_{\partial \Omega}=0 .
\end{array}\right.
$$

Applying the general results in [6], we know that both (5.6) and (5.7) are global well-posed in $\mathcal{H}$, and $\left\{D_{\varepsilon}(t)\right\}_{t \geq 0}$ also forms a semigroup.

Moreover, as in $\S 3$, we can deduce a similar estimate for $\left\{D_{\varepsilon}(t)\right\}_{t \geq 0}$ in $\mathcal{H}$, and so $\left\{K_{\varepsilon}(t)\right\}_{t \geq 0}$ (from (5.5)): There exist constants $C_{M}$ and $\Lambda_{1}$ such that for any $\varepsilon \in[0,1]$ and any $\left(u_{0}, v_{0}\right) \in B_{0}$,

$$
\left\|D_{\varepsilon}(t) B_{0}\right\|_{\mathcal{H}}^{2}+\left\|v_{t t}(t)\right\|^{2}+\varepsilon\left\|\nabla v_{t t}(t)\right\|^{2}+\int_{T_{1 B}+2}^{t}\left\|\nabla v_{t t}(s)\right\|^{2} d s \leq \bar{M} \text { as } t \geq \Lambda_{1}
$$

and

$$
\forall \varepsilon \in[0,1],\left\|D_{\varepsilon}(t) B_{0}\right\|_{\mathcal{H}}^{2}+\left\|K_{\varepsilon}(t) B_{0}\right\|_{\mathcal{H}}^{2} \leq C_{M} \text { for all } t \geq 0 .
$$

5.2. The first a priori estimate. We begin with the decay estimate for the solution of (5.6).

Lemma 5.1. There exist a constant $k>0$ and $Q(\cdot) \in \mathfrak{J}$ such that

$$
\left\|D_{\varepsilon}(t) B_{0}\right\|_{\mathcal{H}}^{2} \leq Q\left(\left\|B_{0}\right\|_{\mathcal{H}}\right) e^{-k t} \quad \text { for all } t \geq 0 \text { and any } \varepsilon \in[0,1] \text {, }
$$

where both $k$ and $Q(\cdot)$ are independent of $\varepsilon \in[0,1]$.

Proof. Multiplying (5.6) by $v_{t}+\theta v$, we have

$$
\frac{d}{d t} E_{3 v}(t)+2\left(\theta\|\nabla v\|^{2}+\left\|\nabla v_{t}\right\|^{2}+\theta \int_{\Omega} v f_{0}(v) d x\right)=2\left\langle v_{t}+\theta v, \theta v_{t}-\varepsilon \theta \Delta v_{t}\right\rangle,
$$

where $E_{3 v}(t)=\left\|v_{t}+\theta v\right\|^{2}+(1+\theta)\|\nabla v\|^{2}+\varepsilon\left\|\nabla\left(v_{t}+\theta v\right)\right\|^{2}+2 \int_{\Omega} F_{0}(v) d x$ and $F_{0}(v)=\int_{0}^{v} f_{0}(s) d s$.

Then, using (5.2), we have

$$
\begin{aligned}
& \frac{1}{2}\left\|v_{t}\right\|^{2}+\left(1+\theta-\theta^{2}-\frac{\theta^{2}}{\lambda_{1}}\right)\|\nabla v\|^{2}+\varepsilon\left\|\nabla v_{t}\right\|^{2} \\
& \quad \leq E_{3 v}(t) \leq 2\left\|v_{t}\right\|^{2}+2 \varepsilon\left\|\nabla v_{t}\right\|^{2}+C_{\theta, \lambda_{1}}\|\nabla v\|^{2}+2 \int_{\Omega} F_{0}(v) d x
\end{aligned}
$$


and

$$
\left|2\left\langle v_{t}+\theta v, \theta v_{t}-\varepsilon \theta \Delta v_{t}\right\rangle\right| \leq 2 \theta\left(\left\|v_{t}\right\|^{2}+\left\|\nabla v_{t}\right\|^{2}+\theta\|v\|\left\|v_{t}\right\|+\theta\left\|\nabla v_{t}\right\|\left\|\nabla v_{t}\right\|\right) .
$$

Note that, from (5.1) and (5.9), we have

$$
0 \leq \int_{\Omega} F_{0}(v) d x \leq C\left(\|v\|^{2}+\|v\|^{\frac{2 N}{N-2}}\right) \leq C_{M}\|\nabla v\|^{2} .
$$

Hence, by taking $\theta$ small enough, we can deduce from (5.10) that

$$
\frac{d}{d t} E_{3 v}(t)+C_{M, \lambda_{1}, \theta} E_{3 v}(t) \leq 0
$$

where the constant $C_{M, \lambda_{1}, \theta}$ depends on $M, \lambda_{1}$ and $\theta$, but not on $\varepsilon$, which, combining with (5.11) and (5.13), implies that

$$
\begin{aligned}
& \frac{1}{2}\left\|v_{t}(t)\right\|^{2}+\left(1+\theta-\theta^{2}-\frac{\theta^{2}}{\lambda_{1}}\right)\|\nabla v(t)\|^{2}+\varepsilon\left\|\nabla v_{t}(t)\right\|^{2} \\
& \quad \leq e^{-C_{M, \lambda_{1}, \theta} t}\left(\frac{4}{\lambda_{1}}\left\|\nabla v_{t}(0)\right\|^{2}+\left(1+\theta+2 \theta^{2}+2 C_{M}\right)\|\nabla v(0)\|^{2}\right) .
\end{aligned}
$$

Now, to complete our proof, we multiply (5.6) by $v_{t}$ and obtain

$$
\begin{aligned}
\left\|\nabla v_{t}\right\|^{2} & \leq-\left\langle v_{t t}, v_{t}\right\rangle+\left\langle\Delta v, v_{t}\right\rangle+\varepsilon\left\langle\Delta v_{t t}, v_{t}\right\rangle-\left\langle f_{0}(v), v_{t}\right\rangle \\
& \leq\left\|v_{t t}\right\|\left\|v_{t}\right\|+\|\nabla v\|\left\|\nabla v_{t}\right\|+\varepsilon\left\|\nabla v_{t t}\right\|\left\|\nabla v_{t}\right\|+C\|v\|\left\|v_{t}\right\|+C\|\nabla v\|^{N-2}\left\|\nabla v_{t}\right\|,
\end{aligned}
$$

which, combining with (5.8)-(5.9), implies that

$$
\left\|\nabla v_{t}\right\|^{2} \leq 2 \sqrt{\bar{M}}\left(\left\|v_{t}\right\|+\sqrt{\varepsilon}\left\|\nabla v_{t}\right\|\right)+C_{\lambda_{1}, M}\|\nabla v\|^{2} \quad \text { as } t \geq \Lambda_{1} .
$$

Therefore, combining with the estimates (5.14), we can finally deduce that

$$
\left\|\nabla v_{t}\right\|^{2} \leq C_{\bar{M}, M, \lambda_{1}, \theta} e^{-\frac{C_{M, \lambda_{1}, \theta}}{2} t}+C_{\lambda_{1}, M}\|\nabla v\|^{2} \text { as } t \geq \Lambda_{1},
$$

which, combining with (5.14) again for the estimate of $\|\nabla v(t)\|^{2}$ and using Lemma 5.2 below with (5.9), allows us to complete our proof by taking $k=\frac{C_{M, \lambda_{1}, \theta}}{2}$ and some increasing function $Q(\cdot)$.

Lemma 5.2. Let $\{S(t)\}_{t \geq 0}$ be a continuous semigroup on the Banach space $X$, satisfying

$$
\|S(t) B\|_{X} \leq Q_{1}\left(\|B\|_{X}\right) e^{-\mu t} \text { as } t \geq t_{0} \text {, and }\|\{S(t) B: t \geq 0\}\|_{X} \leq Q_{2}\left(\|B\|_{X}\right) .
$$

Then

$$
\|S(t) B\|_{X} \leq Q_{3}\left(\|B\|_{X}, t_{0}\right) e^{-\mu t} \quad \text { for all } t \geq 0 .
$$

Its proof is obvious and we omit it here.

The next estimate is about the solution of (5.7):

Lemma 5.3. There exist $k_{1}>0$ and $Q(\cdot) \in \mathfrak{J}$ such that for any $t \geq 0$ and any $\varepsilon \in[0,1]$,

$$
\left\|K_{\varepsilon}(t) B_{0}\right\|_{\mathcal{H}_{\varepsilon}^{\sigma}}^{2} \leq Q\left(\left\|B_{0}\right\|_{\mathcal{H}}\right) e^{k_{1} t},
$$

where both $k_{1}$ and $Q(\cdot)$ are independent of $\varepsilon \in[0,1]$, and $\sigma=\frac{1}{2} \min \left\{1, \frac{N}{2}-1\right\}$.

Proof. Multiplying (5.7) by $A^{\sigma} w_{t}(t)$ (recall that $A=-\Delta$ ), then the proof is the same as that in [28, Lemma 4.2]. 
Based on Lemma [5.1] and Lemma [5.3, following the idea in Zelik [37, we can now decompose $u(t)$ as follows (the proof is completely similar to that in [28, 35, 37. since the estimates in Lemmas 5.1 and 5.3 hold uniformly w.r.t. $\varepsilon \in[0,1])$ :

Lemma 5.4. Let $\left(u(t), u_{t}(t)\right)$ be the solution of $E_{\varepsilon}$ corresponding to the initial data $\left(u_{0}, v_{0}\right) \in B_{0}$. Then, for any $\eta>0$, we can decompose $\left(u(t), u_{t}(t)\right)=$ $S_{\varepsilon}(t)\left(u_{0}, v_{0}\right)$ as

$$
u(t)=v_{1}(t)+w_{1}(t), \quad \text { for all } t \geq 0,
$$

where $v_{1}(t)$ and $w_{1}(t)$ satisfy the following estimates:

$$
\int_{s}^{t}\left\|\nabla v_{1}(\tau)\right\|^{2} d \tau \leq \eta(t-s)+C_{\eta} \text { for all } t \geq s \geq 0
$$

and

$$
\left\|A^{\frac{1+\sigma}{2}} w_{1}(t)\right\|^{2} \leq K_{\eta} \text { for all } t \geq 0
$$

with the constants $C_{\eta}$ and $K_{\eta}$ depending on $\eta,\left\|B_{0}\right\|_{\mathcal{H}}$ and $\|g\|$, but both are independent of $\varepsilon \in[0,1]$.

5.3. The second a priori estimate. The main purpose of this subsection is to deduce some uniformly asymptotic (w.r.t. $\varepsilon \in[0,1]$ and time $t$ ) a priori estimates about the solution of $E_{\varepsilon}$.

Lemma 5.5. There exist positive constants $\bar{\nu}, \bar{R}>0$ and $Q_{1}(\cdot) \in \mathfrak{J}$ such that for each $\varepsilon \in[0,1]$, there is a subset $\bar{B}_{\varepsilon} \subset \mathcal{H}_{\varepsilon}^{1}$ satisfying

$$
\left\|\bar{B}_{\varepsilon}\right\|_{\mathcal{H}_{\varepsilon}^{1}}^{2}=\sup _{(u, v) \in \bar{B}_{\varepsilon}}\left\{\|\Delta u\|^{2}+\|\nabla v\|^{2}+\varepsilon\|\Delta v\|^{2}\right\} \leq \bar{R}
$$

and the exponential attraction

$$
\operatorname{dist}_{\mathcal{H}_{\varepsilon}^{0}}\left(S_{\varepsilon}(t) B_{0}, \bar{B}_{\varepsilon}\right) \leq Q_{1}\left(\left\|B_{0}\right\|_{\mathcal{H}}\right) e^{-\bar{\nu} t} \quad \text { for all } t \geq 0 .
$$

Here, all $\bar{\nu}, \bar{R}$ and $Q_{1}(\cdot)$ are independent of $\varepsilon \in[0,1]$, and $\operatorname{dist}_{\mathcal{H}_{\varepsilon}^{0}}(\cdot, \cdot)$ denotes the Hausdorff semidistance with respect to the $\mathcal{H}_{\varepsilon}^{0}$-norm.

This lemma shows some asymptotic regularity of $\left\{S_{\varepsilon}(t)\right\}_{t \geq 0}$ for each fixed $\varepsilon \in$ $[0,1]$. Combining with the attraction transitivity lemma established in $[15$, Theorem 5.1], there are at least two ways to prove this lemma: one is as that in [28, 35. to apply the idea introduced in Zelik [37]; another one is the method introduced recently in Conti and Pata [13. Here we will use the method in [13.

Proof of Lemma 5.5. It is convenient to separate our proof into three steps. We emphasize especially that all the generic constants in the proof are independent of $\varepsilon$.

Step 1. We first claim that (recall $\sigma=\frac{1}{2} \min \left\{1, \frac{N}{2}-1\right\}$ )

$\exists \nu_{\sigma}, R_{\sigma}>0$ and $Q_{\sigma}(\cdot) \in \mathfrak{J}$ such that for each $\varepsilon \in[0,1]$, there is a subset $\bar{B}_{\sigma, \varepsilon} \subset \mathcal{H}_{\varepsilon}^{\sigma}$ satisfying

$$
\left\|\bar{B}_{\sigma, \varepsilon}\right\|_{\mathcal{H}_{\varepsilon}^{\sigma}}^{2}=\sup _{(u, v) \in \bar{B}_{\sigma, \varepsilon}}\left\{\left\|A^{\frac{1+\sigma}{2}} u\right\|^{2}+\left\|A^{\frac{\sigma}{2}} v\right\|^{2}+\varepsilon\left\|A^{\frac{1+\sigma}{2}} v\right\|^{2}\right\} \leq R_{\sigma}
$$

and the exponential attraction

$$
\operatorname{dist}_{\mathcal{H}_{\varepsilon}^{0}}\left(S_{\varepsilon}(t) B_{0}, \bar{B}_{\sigma, \varepsilon}\right) \leq Q_{\sigma}\left(\left\|B_{0}\right\|_{\mathcal{H}}\right) e^{-\nu_{\sigma} t} \quad \text { for all } t \geq 0 .
$$

We will apply Lemma 3.1 with $X=\mathcal{H}_{\varepsilon}^{0}$ and $V=\mathcal{H}_{\varepsilon}^{\sigma}$ (note that $B_{0} \subset \mathcal{H}_{\varepsilon}^{0}$ for any $\varepsilon \in[0,1])$. 
Based on Lemmas 5.1 and 5.3 , the proof of the above claim is completely similar to that in 13] for a strongly damped wave equation. From (5.1) we can write

$$
f_{0}(s)=s \varphi(s) \quad \text { with }|\varphi(s)| \leq C|s|^{\frac{4}{N-2}} .
$$

For any $x \in B_{0}$ and $y \in \mathcal{H}_{\varepsilon}^{0}, z \in \mathcal{H}_{\varepsilon}^{\sigma}$ satisfying $x=y+z$, we decompose the solution of $\left[E_{\varepsilon}\right)$ as $S_{\varepsilon}(t) x=V_{x}^{\varepsilon}(t) y+U_{x}^{\varepsilon}(t) z$, where

$$
V_{x}^{\varepsilon}(t) y=\left(\bar{v}(t), \bar{v}_{t}(t)\right) \quad \text { and } \quad U_{x}^{\varepsilon}(t) z=\left(\bar{w}(t), \bar{w}_{t}(t)\right),
$$

which uniquely solve the following equations, respectively:

$$
\left\{\begin{array}{l}
\bar{v}_{t t}-\Delta \bar{v}_{t}-\Delta \bar{v}-\varepsilon \Delta \bar{v}_{t t}=h_{1}, \\
\left.\bar{v}\right|_{\partial \Omega}=0,\left(\bar{v}(0), \bar{v}_{t}(0)\right)=y,
\end{array}\right.
$$

and

$$
\left\{\begin{array}{l}
\bar{w}_{t t}-\Delta \bar{w}_{t}-\Delta \bar{w}-\varepsilon \Delta \bar{w}_{t t}=h_{2} \\
\left.\bar{w}\right|_{\partial \Omega}=0,\left(\bar{w}(0), \bar{w}_{t}(0)\right)=z
\end{array}\right.
$$

with

$$
h_{1}=-\bar{v} \varphi(v) \quad \text { and } h_{2}=g(x)-f(u)+\bar{v} \varphi(v),
$$

and $v(t)$ is the solution of (5.6) corresponding to the initial data $x$.

From (5.1), (5.3), (5.17) and Lemmas 5.1, 5.3, we can directly calculate that

$$
\left\|h_{1}\right\|_{L^{\frac{2 N}{N+2}}} \leq C\|\nabla \bar{v}\|\|\nabla v\|^{\frac{4}{N-2}} \leq C_{M} e^{-k^{\prime} t}\|\nabla \bar{v}\|
$$

and similarly

$$
\left\|h_{2}\right\|_{L^{\frac{2 N}{N+2(1-\sigma)}}} \leq C_{M} e^{-k^{\prime} t}\left\|A^{\frac{1+\sigma}{2}} \bar{w}\right\|+C_{M} e^{k_{1}^{\prime} t},
$$

where we only have used the embedding $H_{0}^{1}(\Omega) \hookrightarrow L^{\frac{2 N}{N-2}}(\Omega), D\left(A^{\frac{1+\sigma}{2}}\right) \hookrightarrow$ $L^{\frac{2 N}{N-2(1+\sigma)}}(\Omega)$ and $D\left(A^{\frac{1-\sigma}{2}}\right) \hookrightarrow L^{\frac{2 N}{N-2(1-\sigma)}}(\Omega)$ (which are independent of $\varepsilon$ ).

Hence, multiplying (5.18) and (5.19) respectively by $\bar{v}_{t}+\theta \bar{v}$ and $A^{\sigma}\left(\bar{w}_{t}+\theta \bar{w}\right)$, through some similar calculations as that in the proof of Lemma 4.1 (see also the proof of 13, Theorem 4.3]), we can verify that all the conditions of Lemma 3.1] are satisfied for the case $X=\mathcal{H}_{\varepsilon}^{0}, V=\mathcal{H}_{\varepsilon}^{\sigma}$ and $T(t)=S_{\varepsilon}(t)$. Moreover, since there is a $c_{1}>0$ (independent of $\varepsilon$ ) such that $c_{1}\left\|B_{0}\right\|_{\mathcal{H}} \geq\left\|B_{0}\right\|_{\mathcal{H}_{\varepsilon}^{0}}$ for any $\varepsilon \in[0,1]$ and the constants in our estimates are all independent of $\varepsilon$, consequently, $\nu_{\sigma}, R_{\sigma}$ and $Q_{\sigma}(\cdot)$ are all independent of $\varepsilon \in[0,1]$, we can then deduce our claim.

Step 2. We claim that

there exists a positive constant $\bar{R}_{\sigma}$ which depends only on $R_{\sigma}$ such that

$$
\forall \varepsilon \in[0,1],\left\|S_{\varepsilon}(t) \bar{B}_{\sigma, \varepsilon}\right\|_{\mathcal{H}_{\varepsilon}^{\sigma}}^{2} \leq \bar{R}_{\sigma} \quad \text { for all } t \geq 0 .
$$

This claim can be proved completely similar to that in [28, Lemma 4.5] via multiplying $\left(E_{\varepsilon}\right)$ by $A^{\sigma}\left(u_{t}+\theta u\right)$, and applying Lemma 5.4 to overcome the critical nonlinearity.

Step 3. Based on Steps 1 and 2, applying the attraction transitivity lemma given in [15. Theorem 5.1] and noticing the Hölder continuity Lemma 4.4, we can prove our lemma by performing a bootstrap argument, whose proof is now simple since Step 1 makes the nonlinear term become subcritical to some extent (e.g., see [29] for some similar calculations). 
5.4. Proof of Theorem 2.1. Lemma 5.5 has shown some asymptotic regularity; however, the radius of $\left\|\bar{B}_{\varepsilon}\right\|_{\mathcal{H}^{1}}$ depends on $\varepsilon$ and the distance only under the $\mathcal{H}_{\varepsilon}^{0}$ norm.

To prove Theorem 2.1, we first give two lemmas as preliminary.

Lemma 5.6. There exists $R_{1}>0$ such that for any bounded (in $\mathcal{H}_{\varepsilon}^{1}$ ) subset $B \subset$ $\mathcal{H}_{\varepsilon}^{1}$, there is a $T_{1}=T_{1}\left(\|B\|_{\mathcal{H}_{\varepsilon}^{1}}\right)$ such that

$$
\forall \varepsilon \in[0,1],\left\|S_{\varepsilon}(t) B\right\|_{\mathcal{H}_{\varepsilon}^{1}}^{2} \leq R_{1} \quad \text { for all } t \geq T_{1} .
$$

Proof. Multiplying $\left(E_{\varepsilon}\right)$ by $-\Delta\left(u_{t}+\theta u\right)$ and taking $\theta$ small enough, we have

$$
\begin{aligned}
& \frac{d}{d t}\left(\left\|\nabla\left(u_{t}+\theta u\right)\right\|^{2}+(1+\theta)\|\Delta u\|^{2}+\varepsilon\left\|\Delta\left(u_{t}+\theta u\right)\right\|^{2}\right)+C_{\theta}\left(\left\|\Delta u_{t}\right\|^{2}+\|\Delta u\|^{2}\right) \\
& \leq C_{\theta}\|g\|^{2}+C \int_{\Omega}\left(1+|u|^{\frac{4}{N-2}}\right)|\nabla u|\left(\left|\nabla u_{t}\right|+|\nabla u|\right) .
\end{aligned}
$$

Then, as that in [29, Lemma 5.5], applying Lemma 5.4 we can deal with the nonlinear term and finally complete the proof as an application of the Gronwall inequality.

Lemma 5.7. There exists $R_{2}>0$ such that for any bounded (in $\mathcal{H}_{\varepsilon}^{1}$ ) subset $B \subset$ $\mathcal{H}_{\varepsilon}^{1}$, there is a $T_{2}=T_{2}\left(\|B\|_{\mathcal{H}_{\varepsilon}^{1}}\right)$ such that

$$
\forall \varepsilon \in[0,1],\left\|S_{\varepsilon}(t) B\right\|_{\mathcal{H}^{1}}^{2} \leq R_{2} \quad \text { for all } t \geq T_{2} .
$$

Proof. From Lemma 5.6 above, we only need to estimate that the bound of $\left\|\Delta u_{t}\right\|^{2}$ is independent of $\varepsilon \in[0,1]$.

Multiplying $\left(E_{\varepsilon}\right)$ by $-\Delta u_{t t}$, we have

$$
\frac{1}{2} \frac{d}{d t} E_{4 u}(t)+\left\|\nabla u_{t t}\right\|^{2}+\varepsilon\left\|\Delta u_{t t}\right\|^{2}-\left\|\Delta u_{t}\right\|^{2}=-\left\langle f^{\prime}(u) u_{t}, \Delta u_{t}\right\rangle,
$$

where $E_{4 u}(t)=\left\|\Delta u_{t}\right\|^{2}+2\left\langle\Delta u, \Delta u_{t}\right\rangle+2\left\langle\Delta u_{t}, g\right\rangle-2\left\langle f(u), \Delta u_{t}\right\rangle$.

Note that, as $t \geq T_{1}\left(\|B\|_{\mathcal{H}_{\varepsilon}^{1}}\right)$ (given in Lemma [5.6) , we have

$$
\begin{aligned}
\left|\left\langle f^{\prime}(u) u_{t}, \Delta u_{t}\right\rangle\right| & \leq C \int_{\Omega}\left(1+|u|^{\frac{4}{N-2}}\right)\left|u_{t} \| \Delta u_{t}\right| d x \\
& \leq C\left(\left\|u_{t}\right\|+\|\nabla u\|\left\|\Delta u_{t}\right\|\right)\left\|\Delta u_{t}\right\| \leq C_{\lambda_{1}, R_{1}}\left\|\Delta u_{t}\right\|^{2}
\end{aligned}
$$

and

$$
\begin{aligned}
\frac{1}{4}\left\|\Delta u_{t}(t)\right\|^{2}-4\left(\|\Delta u\|^{2}+\|g\|^{2}+\|f(u)\|^{2}\right) & \leq E_{4 u}(t) \\
& \leq 4\left\|\Delta u_{t}(t)\right\|^{2}+\|\Delta u\|^{2}+\|g\|^{2}+\|f(u)\|^{2} .
\end{aligned}
$$

At the same time, applying Lemma [5.6] again and integrating (5.23) on $[t, t+1]$ yield

$$
\int_{t}^{t+1}\left\|\Delta u_{t}(s)\right\|^{2} d s \leq C_{R_{1}} \text { for all } t \geq T_{1}\left(\|B\|_{\mathcal{H}_{\varepsilon}^{1}}\right) .
$$

Hence, we can complete our proof by applying the uniform Gronwall lemma to (5.24).

Now, we are ready to prove Theorem 2.1 . 
Proof of Theorem 2.1. Set

$$
\mathbb{B}=\left\{z \in \mathcal{H}^{1}:\|z\|_{\mathcal{H}^{1}}^{2} \leq R_{2}\right\},
$$

where the constant $R_{2}$ comes from Lemma 5.7 above.

From Lemmas 5.7 and 5.5. we know that there is a $t_{0}$ such that $S_{\varepsilon}(t) \bar{B}_{\varepsilon} \subset \mathbb{B}$ (recall that $\bar{B}_{\varepsilon}$ is given in (5.15)) for all $t \geq t_{0}$ and any $\varepsilon \in[0,1]$.

On the other hand, note that

$$
c_{1}\|\cdot\|_{\mathcal{H}_{\varepsilon}^{0}} \leq\|\cdot\|_{\mathcal{H}} \leq c_{2}\|\cdot\|_{\mathcal{H}_{\varepsilon}^{1}} \text { for all } \varepsilon \in[0,1]
$$

where $c_{1}, c_{2}>0$ are independent of $\varepsilon$. Then, from Lemma 4.4, there exists $t_{1}$ which depends only on $\left\|B_{0}\right\|_{\mathcal{H}}$ and $\left\|\bar{B}_{\varepsilon}\right\|_{\mathcal{H}_{\varepsilon}^{1}}$ (so only on $M, \bar{R}$ ) such that

$\forall \varepsilon \in[0,1],\left\|S_{\varepsilon}(t) z_{1}-S_{\varepsilon}(t) z_{2}\right\|_{\mathcal{H}} \leq e^{C_{\bar{R}} t}\left\|z_{1}-z_{2}\right\|_{\mathcal{H}_{\varepsilon}^{0}}^{\frac{1}{2}}, \forall t \geq t_{1}, z_{1} \in B_{0}, z_{2} \in \bar{B}_{\varepsilon}$

and

$$
\forall \varepsilon \in[0,1], S_{\varepsilon}(t) B_{0} \subset B_{0} \quad \text { for all } t \geq t_{1} .
$$

Therefore, from Lemma 5.5. we have

$$
\begin{aligned}
\operatorname{dist}_{\mathcal{H}}\left(S_{\varepsilon}\left(t+t_{0}+t_{1}\right) B_{0}, \mathbb{B}\right) & \leq \operatorname{dist}_{\mathcal{H}}\left(S_{\varepsilon}\left(t+t_{0}+t_{1}\right) B_{0}, S_{\varepsilon}\left(t_{0}+t_{1}\right) \bar{B}_{\varepsilon}\right) \\
& \leq C_{M, \bar{R}, t_{0}+t_{1}} \operatorname{dist}_{\mathcal{H}_{\varepsilon}^{0}}^{\frac{1}{2}}\left(S_{\varepsilon}(t) B_{0}, \bar{B}_{\varepsilon}\right)(\text { by }(5.27)) \\
& \leq C_{M, \bar{R}, t_{0}+t_{1}} \sqrt{Q_{1}\left(\left\|B_{0}\right\|_{\mathcal{H}}\right)} e^{-\frac{\bar{\nu}}{2} t}, \quad \forall t \geq 0 .
\end{aligned}
$$

Hence, noting that $t_{0}, t_{1}$ and $\bar{R}$ are all fixed, we can complete the proof by taking $\nu=\frac{\bar{\nu}}{2}$ and applying Lemma 5.2

5.5. Applications of Theorem 2.1. As the application of Theorem 2.1, in this subsection, we consider the existence of finite dimensional exponential attractors and the upper semicontinuity of global attractors.

5.5.1. A priori estimates. For the subset $\mathbb{B}$ defined in (5.25), from Lemma 4.1 and Lemma 4.3 we know that there is a $t_{\mathbb{B}}$ such that

$\forall \varepsilon \in[0,1],\left\|\nabla u_{t}(t)\right\|^{2}+\left\|u_{t t}\right\|^{2}+\varepsilon\left\|\nabla u_{t t}(t)\right\|^{2} \leq M+M_{4}$ for all $t \geq t_{\mathbb{B}},\left(u_{0}, v_{0}\right) \in \mathbb{B}$, where $\left(u(t), u_{t}(t)\right)=S_{\varepsilon}(t)\left(u_{0}, v_{0}\right)$.

Now, for each $\varepsilon \in[0,1]$, define $\hat{B}_{\varepsilon}$ as follows:

$$
\hat{B}_{\varepsilon}=\bigcup_{t \geq t_{\mathbb{B}}+T_{2}} S_{\varepsilon}(t) \mathbb{B},
$$

where $T_{2}$ is the time given in Lemma 5.7 corresponding to $\mathbb{B}$. Then, for each $\varepsilon \in[0,1]$ we have that $\hat{B}_{\varepsilon}$ is positive invariant under $S_{\varepsilon}(t)$ (i.e., $S_{\varepsilon}(t) \hat{B}_{\varepsilon}=\hat{B}_{\varepsilon}$, $\forall t \geq 0$ ) and (from Lemma 5.7)

$$
\forall \varepsilon \in[0,1],\left\|\hat{B}_{\varepsilon}\right\|_{\mathcal{H}^{1}}^{2} \leq R_{2} .
$$

Moreover, we have the following results:

Lemma 5.8. There exists a $\mathcal{T}>0$ such that for every $\varepsilon \in[0,1]$, the semigroup $S_{\varepsilon}(t)$ satisfies the following properties: $S_{\varepsilon}(\mathcal{T})$ admits a decomposition of the form

$$
S_{\varepsilon}(\mathcal{T})=L_{\varepsilon}+N_{\varepsilon}, \quad L_{\varepsilon}: \hat{B}_{\varepsilon} \rightarrow \mathcal{H}_{\varepsilon}^{0}, \quad N_{\varepsilon}: \hat{B}_{\varepsilon} \rightarrow \mathcal{H}_{\varepsilon}^{\gamma},
$$


where $L_{\varepsilon}$ and $N_{\varepsilon}$ satisfy the estimates

$$
\left\|L_{\varepsilon}\left(z_{1}\right)-L_{\varepsilon}\left(z_{2}\right)\right\|_{\mathcal{H}_{\varepsilon}^{0}} \leq \frac{1}{4}\left\|z_{1}-z_{2}\right\|_{\mathcal{H}_{\varepsilon}^{0}}, \quad \forall z_{1}, z_{2} \in \hat{B}_{\varepsilon}
$$

and

$$
\left\|N_{\varepsilon}\left(z_{1}\right)-N_{\varepsilon}\left(z_{2}\right)\right\|_{\mathcal{H}_{\varepsilon}^{\gamma}} \leq C_{R_{2}, \mathcal{T}}\left\|z_{1}-z_{2}\right\|_{\mathcal{H}_{\varepsilon}^{0}}, \quad \forall z_{1}, z_{2} \in \hat{B}_{\varepsilon}
$$

with the constant $C_{R_{2}, \mathcal{T}}$ which is independent of $\varepsilon$ and

$$
\gamma= \begin{cases}1, & N=3,4,5,6 \\ \frac{4}{N-2}, & N>6\end{cases}
$$

Proof. For any two initial data $z_{i} \in \hat{B}_{\varepsilon}$ with solution $S_{\varepsilon}(t) z_{i}=\left(u^{i}(t), u_{t}^{i}(t)\right)(i=$ $1,2)$, we decompose the difference $S_{\varepsilon}(t) z_{1}-S_{\varepsilon}(t) z_{2}$ as follows:

$$
S_{\varepsilon}(t) z_{1}-S_{\varepsilon}(t) z_{2}=L_{\varepsilon}(t)\left(z_{1}-z_{2}\right)+N_{\varepsilon}(t)\left(z_{1}-z_{2}\right),
$$

where $L_{\varepsilon}(t)\left(z_{1}-z_{2}\right)=\left(\tilde{v}(t), \tilde{v}_{t}(t)\right)$ solves

$$
\left\{\begin{array}{l}
\tilde{v}_{t t}-\Delta \tilde{v}_{t}-\Delta \tilde{v}-\varepsilon \Delta \tilde{v}_{t t}=0, \\
\left(\tilde{v}(0), \tilde{v}_{t}(0)\right)=z_{1}-z_{2},\left.\tilde{v}\right|_{\partial \Omega}=0,
\end{array}\right.
$$

and $N_{\varepsilon}(t)\left(z_{1}-z_{2}\right)=\left(\tilde{w}(t), \tilde{w}_{t}(t)\right)$ solves

$$
\left\{\begin{array}{l}
\tilde{w}_{t t}-\Delta \tilde{w}_{t}-\Delta \tilde{w}-\varepsilon \Delta \tilde{w}_{t t}+f\left(u^{1}\right)-f\left(u^{2}\right)=0, \\
\left(\tilde{w}(0), \tilde{w}_{t}(0)\right)=(0,0),\left.\tilde{w}\right|_{\partial \Omega}=0 .
\end{array}\right.
$$

In the following, for clarity, we decompose the remainder proof into two steps.

Step 1. Similar to the proof of Lemma 4.4 for (5.34) we can deduce that

$$
\left\|L_{\varepsilon}(t) z_{1}-L_{\varepsilon}(t) z_{2}\right\|_{\mathcal{H}_{\varepsilon}^{0}}^{2}=\left\|\left(\tilde{v}(t), \tilde{v}_{t}(t)\right)\right\|_{\mathcal{H}_{\varepsilon}^{0}}^{2} \leq Q\left(\left\|\hat{B}_{\varepsilon}\right\|_{\mathcal{H}}\right)\left\|z_{1}-z_{2}\right\|_{\mathcal{H}_{\varepsilon}^{0}}^{2} e^{-\mu_{1} t},
$$

where the constant $\mu_{1}$ only depends on the first eigenvalue $\lambda_{1}$. Hence, by taking $T^{\prime}>0$ large enough, we have

$$
\left\|L_{\varepsilon}\left(t+T^{\prime}\right) z_{1}-L_{\varepsilon}\left(t+T^{\prime}\right) z_{2}\right\|_{\mathcal{H}_{\varepsilon}^{0}} \leq \frac{1}{4}\left\|z_{1}-z_{2}\right\|_{\mathcal{H}_{\varepsilon}^{0}} \text { for all } t \geq 0 .
$$

Step 2. For $\tilde{w}(t)$, multiplying (5.35) by $A^{\gamma} \tilde{w}_{t}(t)$ (where $\gamma$ is given in (5.33)) we obtain that

$$
\begin{aligned}
& \frac{1}{2} \frac{d}{d t}\left(\left\|A^{\frac{\gamma}{2}} \tilde{w}_{t}\right\|^{2}+\left\|A^{\frac{1+\gamma}{2}} \tilde{w}\right\|^{2}+\varepsilon\left\|A^{\frac{1+\gamma}{2}} \tilde{w}_{t}\right\|^{2}\right)+\left\|A^{\frac{1+\gamma}{2}} \tilde{w}_{t}\right\|^{2} \\
& \quad+\left\langle f\left(u^{1}\right)-f\left(u^{2}\right), A^{\gamma} \tilde{w}_{t}\right\rangle=0 .
\end{aligned}
$$

Case 1. $N=3,4$. Then using the embedding $D(A) \hookrightarrow L^{p}(\Omega)$ for any $p \geq 1$, we have

$$
\begin{aligned}
\left|\left\langle f\left(u^{1}\right)-f\left(u^{2}\right), A \tilde{w}_{t}\right\rangle\right| & \leq C\left(1+\left\|u^{1}\right\|_{H^{2}}^{\frac{4}{N-2}}+\left\|u^{2}\right\|_{H^{2}}^{\frac{4}{N-2}}\right)\left\|\nabla\left(u^{1}-u^{2}\right)\right\|\left\|A \tilde{w}_{t}\right\| \\
& \leq C_{R_{2}}\left\|\nabla\left(u^{1}-u^{2}\right)\right\|\left\|A \tilde{w}_{t}\right\| \\
& \leq C_{R_{2}} e^{C_{R_{2}} t}\left\|z_{1}-z_{2}\right\|\left\|_{\mathcal{H}_{\varepsilon}^{0}}\right\| A \tilde{w}_{t} \| \\
& \leq C_{R_{2}, t}\left\|z_{1}-z_{2}\right\|_{\mathcal{H}_{\varepsilon}^{0}}^{2}+\frac{1}{2}\left\|A \tilde{w}_{t}\right\|^{2},
\end{aligned}
$$

where we have used (5.30) and (4.21). 
Case 2. $N=5$,6. Since $\frac{4 N}{N-2} \leq \frac{2 N}{N-4}$ and embedding $D(A) \hookrightarrow L^{\frac{2 N}{N-4}}(\Omega)$, we also have

$$
\begin{aligned}
\left|\left\langle f\left(u^{1}\right)-f\left(u^{2}\right), A \tilde{w}_{t}\right\rangle\right| & \leq C\left(1+\left\|u^{1}\right\|_{H^{2}}^{\frac{4}{N-2}}+\left\|u^{2}\right\|_{H^{2}}^{\frac{4}{N-2}}\right)\left\|\nabla\left(u^{1}-u^{2}\right)\right\|\left\|A \tilde{w}_{t}\right\| \\
& \leq C_{R_{2}, t}\left\|z_{1}-z_{2}\right\|_{\mathcal{H}_{\varepsilon}^{0}}^{2}+\frac{1}{2}\left\|A \tilde{w}_{t}\right\|^{2} .
\end{aligned}
$$

Case 3. $N>6$. Noting that $1=\frac{N-2}{2 N}+\frac{N-2(1-\gamma)}{2 N}+\frac{4-2 \gamma}{2 N}$ and $\frac{4}{N-2} \cdot \frac{2 N}{4-2 \gamma}=\frac{2 N}{N-4}$, we have

$$
\begin{aligned}
\left|\left\langle f\left(u^{1}\right)-f\left(u^{2}\right), A^{\gamma} \tilde{w}_{t}\right\rangle\right| & \leq C\left(1+\left\|u^{1}\right\|_{H^{2}}^{\frac{4}{N-2}}+\left\|u^{2}\right\|_{H^{2}}^{\frac{4}{N-2}}\right)\left\|\nabla\left(u^{1}-u^{2}\right)\right\|\left\|A^{\frac{1+\gamma}{2}} \tilde{w}_{t}\right\| \\
& \leq C_{R_{2}, t}\left\|z_{1}-z_{2}\right\|_{\mathcal{H}_{\varepsilon}^{0}}^{2}+\frac{1}{2}\left\|A^{\frac{1+\gamma}{2}} \tilde{w}_{t}\right\|^{2} .
\end{aligned}
$$

Therefore, for any $N \geq 3$, we have

$$
\begin{aligned}
& \frac{d}{d t}\left(\left\|A^{\frac{\gamma}{2}} \tilde{w}_{t}\right\|^{2}+\left\|A^{\frac{1+\gamma}{2}} \tilde{w}\right\|^{2}+\varepsilon\left\|A^{\frac{1+\gamma}{2}} \tilde{w}_{t}\right\|^{2}\right)+\left\|A^{\frac{1+\gamma}{2}} \tilde{w}_{t}\right\|^{2} \\
& \quad \leq 2 C_{R_{2}, t}\left\|z_{1}-z_{2}\right\|_{\mathcal{H}_{\varepsilon}^{0}}^{2} \quad \text { for all } t \geq 0,
\end{aligned}
$$

which, noting that $\left(\tilde{w}(0), \tilde{w}_{t}(0)\right)=(0,0)$, implies that

$$
\begin{aligned}
\left\|\tilde{w}(t), \tilde{w}_{t}(t)\right\|_{\mathcal{H}_{\varepsilon}^{\gamma}}^{2} & =\left\|A^{\frac{\gamma}{2}} \tilde{w}_{t}(t)\right\|^{2}+\left\|A^{\frac{1+\gamma}{2}} \tilde{w}(t)\right\|^{2}+\varepsilon\left\|A^{\frac{1+\gamma}{2}} \tilde{w}_{t}(t)\right\|^{2} \\
& \leq C_{R_{2}, t}\left\|z_{1}-z_{2}\right\|_{\mathcal{H}_{\varepsilon}^{0}}^{2} \quad \text { for all } t \geq 0 .
\end{aligned}
$$

Hence, taking

$$
\mathcal{T}=T^{\prime} \quad \text { and } L_{\varepsilon}=L_{\varepsilon}(\mathcal{T}), \quad N_{\varepsilon}=N_{\varepsilon}(\mathcal{T})
$$

then, from (5.36) and (5.37), we can see that $\mathcal{T}, L_{\varepsilon}$ and $N_{\varepsilon}$ satisfy (5.31)-(5.32), respectively.

Lemma 5.9. For an arbitrary fixed time $T>0$ and any $\varepsilon \in[0,1]$, the semigroup $S_{\varepsilon}(t)$ is Lipschitz continuous on $[0, T] \times \hat{B}_{\varepsilon}$ in the following sense: there exists a positive constant $\bar{C}_{T, R_{2}}$ such that for any $z_{i} \in \hat{B}_{\varepsilon}, t_{i} \in[0, T], i=1,2$,

$$
\left\|S_{\varepsilon}\left(t_{1}\right) z_{1}-S_{\varepsilon}\left(t_{2}\right) z_{2}\right\|_{\mathcal{H}_{\varepsilon}^{0}} \leq \bar{C}_{T, R_{2}}\left(\left|t_{1}-t_{2}\right|+\left\|z_{1}-z_{2}\right\|_{\mathcal{H}_{\varepsilon}^{0}}\right) .
$$

Proof. Obviously, we have

$$
\left\|S_{\varepsilon}\left(t_{1}\right) z_{1}-S_{\varepsilon}\left(t_{2}\right) z_{2}\right\|_{\mathcal{H}_{\varepsilon}^{0}} \leq\left\|S_{\varepsilon}\left(t_{1}\right) z_{1}-S_{\varepsilon}\left(t_{2}\right) z_{1}\right\|_{\mathcal{H}_{\varepsilon}^{0}}+\left\|S_{\varepsilon}\left(t_{2}\right) z_{1}-S_{\varepsilon}\left(t_{2}\right) z_{2}\right\|_{\mathcal{H}_{\varepsilon}^{0}} .
$$

Note that

$$
\left\|S_{\varepsilon}\left(t_{1}\right) z_{1}-S_{\varepsilon}\left(t_{2}\right) z_{1}\right\|_{\mathcal{H}_{\varepsilon}^{0}}=\left\|\int_{t_{1}}^{t_{2}} \frac{d}{d t} S_{\varepsilon}(t) z_{1} d s\right\|_{\mathcal{H}_{\varepsilon}^{0}} \leq\left|\int_{t_{1}}^{t_{2}}\left\|\frac{d}{d t} S_{\varepsilon}(t) z_{1}\right\|_{\mathcal{H}_{\varepsilon}^{o}} d s\right| .
$$

Then from (5.28) and (5.29) we can deduce

$$
\left\|S_{\varepsilon}\left(t_{1}\right) z_{1}-S_{\varepsilon}\left(t_{2}\right) z_{1}\right\|_{\mathcal{H}_{\varepsilon}^{0}} \leq \sqrt{M+M_{4}}\left|t_{1}-t_{2}\right|,
$$

which, combining with (4.21), implies (5.38) immediately. 
5.5.2. Exponential attractors. Based on the preliminary lemmas given in $§ 5.5 .1$, we are now ready to prove the following result about the existence of exponential attractors.

Lemma 5.10. Under Assumption I, for every $\varepsilon \in[0,1]$, there exists a compact subset $\mathcal{E}_{\varepsilon} \subset \mathcal{H}^{1}$, uniformly bounded in $\mathcal{H}^{1}$, which satisfies the following conditions:

(i) $\mathcal{E}_{\varepsilon}$ is semi-invariant with respect to the semigroup $\left\{S_{\varepsilon}(t)\right\}_{t \geq 0}$, that is,

$$
S_{\varepsilon}(t) \mathcal{E}_{\varepsilon} \subset \mathcal{E}_{\varepsilon} \quad \text { for all } t \geq 0
$$

(ii) the fractal dimension of $\mathcal{E}_{\varepsilon}$ is finite, that is,

$$
\operatorname{dim}_{F}\left(\mathcal{E}_{\varepsilon}, \mathcal{H}\right) \leq \Lambda_{\varepsilon}<\infty, \quad \forall \varepsilon \in[0,1] ;
$$

(iii) for each $\varepsilon \in[0,1], \mathcal{E}_{\varepsilon}$ enjoys a uniform exponential attraction property of the form: for any bounded (in $\mathcal{H}$ ) subset $B \subset \mathcal{H}$,

$$
\operatorname{dist}_{\mathcal{H}}\left(S_{\varepsilon}(t) B, \mathcal{E}_{\varepsilon}\right) \leq Q_{\varepsilon}\left(\|B\|_{\mathcal{H}}\right) e^{-\nu^{\prime} t}, \quad \forall t \geq 0 .
$$

Here, $\Lambda_{\varepsilon}$ and $Q_{\varepsilon}(\cdot)$ may depend on $\varepsilon$, but $\nu^{\prime}$ is independent of $\varepsilon$.

Proof. For each $\varepsilon \in[0,1]$, we know that $\hat{B}_{\varepsilon}$ is invariant and compact in $\mathcal{H}_{\varepsilon}^{0}$. Hence, applying the abstract results established in [9, 14, 22] (or see [23, Lemma 9]), from Lemmas 5.8 and 5.9 we can first construct an exponential attractor on $\hat{B}_{\varepsilon}$ with respect to the $\mathcal{H}_{\varepsilon}^{0}$-norm. Then, we can complete the proof, by using the attraction transitivity lemma given in [15, Theorem 5.1] from Lemma 5.5] and the Hölder continuity (4.22).

Remark 5.11. Indeed, as shown in [9, Proposition 2.7, Corollary 2.8] the upper bounds of the fractal dimension of $\mathcal{E}_{\varepsilon}$ can be specified explicitly only by $N_{\frac{\mathcal{H}_{\varepsilon}}{{ }^{5} C_{R_{2}, \mathcal{T}}}}^{\mathcal{H}_{1}}\left(B^{\mathcal{H}_{\varepsilon}^{\gamma}}(0,1)\right)$ and the constant $C_{R_{2}, \mathcal{T}}$ given in $(15.32)$. Here $N_{r}^{V}(C)$ denotes the smallest number of $r$-balls in $V$ needed to cover $C, B^{\mathcal{H}_{\varepsilon}^{\gamma}}(0,1)$ is the unit ball in $\mathcal{H}_{\varepsilon}^{\gamma}$ and $\gamma$ is given in (5.33).

5.5.3. Upper semicontinuity of global attractors. Since $\mathcal{A}_{\varepsilon} \subset \mathcal{E}_{\varepsilon}$, (ii) of Lemma 5.10 implies that the fractal dimension of the global attractor $\mathcal{A}_{\varepsilon}$ is finite too. Moreover, we have the following upper semicontinuity result of $\mathcal{A}_{\varepsilon}$ at $\varepsilon=0$ :

Lemma 5.12. Under Assumption I, the global attractors $\left\{\mathcal{A}_{\varepsilon}\right\}_{\varepsilon \in[0,1]}$ are upper semicontinuous at $\varepsilon=0$ :

$$
\operatorname{dist}_{\mathcal{H}}\left(\mathcal{A}_{\varepsilon}, \mathcal{A}_{0}\right) \rightarrow 0 \quad \text { as } \varepsilon \rightarrow 0^{+} \text {. }
$$

Since the global attractor $\mathcal{A}_{\varepsilon}$ is strictly invariant, i.e., $S_{\varepsilon}(t)=\mathcal{A}_{\varepsilon}$ for all $t \geq 0$, it is obvious to see that

$$
\bigcup_{\varepsilon \in[0,1]} \mathcal{A}_{\varepsilon} \subset \mathbb{B} \text { and compact in } \mathcal{H} .
$$

Therefore, to apply Lemma 3.2 , we can take $K=c_{\mathcal{H}^{1}}(\mathbb{B})$ and we only need to verify condition (3.1).

Let $\varepsilon \in(0,1]$ and $\left(\hat{u}(t), \hat{u}_{t}(t)\right)=S_{\varepsilon}(t) z_{\varepsilon}$ with $z_{\varepsilon} \in \mathcal{A}_{\varepsilon}$; also let $\left(\hat{v}(t), \hat{v}_{t}(t)\right)=$ $S_{0}(t) z_{0}$ with $z_{0} \in \mathbb{B}$. Denote $\hat{w}(t)=\hat{u}(t)-\hat{v}(t)$. Then $\hat{w}$ solves the following equation:

$$
\left\{\begin{array}{l}
\hat{w}_{t t}-\Delta \hat{w}-\Delta \hat{w}_{t}+f(\hat{u})-f(\hat{v})=\varepsilon \Delta \hat{u}_{t t}, \\
\left(\hat{w}(x, 0), \hat{w}_{t}(x, 0)\right)=z_{\varepsilon}-z_{0},\left.\hat{w}\right|_{\partial \Omega}=0 .
\end{array}\right.
$$


Multiplying (5.40) by $\hat{w}_{t}$, we obtain that

$$
\frac{1}{2} \frac{d}{d t}\left(\left\|\hat{w}_{t}\right\|^{2}+\|\nabla \hat{w}\|^{2}\right)+\left\|\nabla \hat{w}_{t}\right\|^{2}+\left\langle f(\hat{u})-f(\hat{v}), \hat{w}_{t}\right\rangle=-\varepsilon \int_{\Omega} \nabla \hat{u}_{t t} \cdot \nabla \hat{w}_{t} .
$$

Therefore,

$$
\frac{d}{d t}\left(\left\|\hat{w}_{t}\right\|^{2}+\|\nabla \hat{w}\|^{2}\right)+\left\|\nabla \hat{w}_{t}\right\|^{2} \leq C_{\|\mathbb{B}\|_{\mathcal{H}}}\|\nabla \hat{w}\|^{2}+\varepsilon^{2}\left\|\nabla \hat{u}_{t t}\right\|^{2} .
$$

Since $z_{\varepsilon} \in \mathcal{A}_{\varepsilon}$, from (5.28) we have

$$
\varepsilon\left\|\nabla \hat{u}_{t t}(t)\right\|^{2} \leq M+M_{4} \quad \text { for all } t \geq 0 .
$$

Hence, integrating (5.41) over $[0, t]$, we have

$$
\left\|\hat{w}_{t}(t)\right\|^{2}+\|\nabla \hat{w}(t)\|^{2} \leq C_{\|\mathbb{B}\|_{\mathcal{H}}, t}\left(\left\|z_{\varepsilon}-z_{0}\right\|_{H_{0}^{1}(\Omega) \times L^{2}(\Omega)}^{2}+\varepsilon\right) \quad \text { for all } t \geq 0 .
$$

Moreover, we also have

$$
\left\|\nabla \hat{w}_{t}(t)\right\|^{2} \leq\left\|\hat{w}_{t t}\right\|\left\|\hat{w}_{t}\right\|+\|\nabla \hat{w}\|\left\|\nabla \hat{w}_{t}\right\|+\varepsilon\left\|\nabla \hat{u}_{t t}\right\|\left\|\nabla \hat{w}_{t}\right\|+C_{\|\mathbb{B}\|_{\mathcal{H}}}\|\nabla \hat{w}\|\left\|\nabla \hat{w}_{t}\right\| .
$$

Then, from Lemma 4.3 and using (5.42), we know that there is a $t_{1}=t_{1}\left(\|\mathbb{B}\|_{\mathcal{H}}\right)$ (which is independent of $\varepsilon$ ) such that

$$
\left\|\nabla \hat{w}_{t}\left(t_{1}+1\right)\right\|^{2} \leq C_{\|\mathbb{B}\|_{\mathcal{H}}, t_{1}}\left(\left\|z_{\varepsilon}-z_{0}\right\|_{H_{0}^{1}(\Omega) \times L^{2}(\Omega)}+\varepsilon\right),
$$

which, combining with (5.42) again, implies

$$
\text { if } \varepsilon_{n} \rightarrow 0^{+} \text {and } \mathcal{A}_{\varepsilon_{n}} \ni z_{n} \rightarrow z_{0} \text {, then } S_{\varepsilon_{n}}\left(t_{1}+1\right) z_{n} \rightarrow S_{0}\left(t_{1}+1\right) z_{0} .
$$

Proof of Lemma 5.12, From (5.39) and (5.43), the proof is a direct application of Lemma 3.2 .

\section{PART II: $g(x) \in H^{-1}$}

Throughout this section, we always assume that Assumption II holds.

We first recall a simple result (its proof can be found in [30]) about an elliptic equation:

Lemma 6.1. Let $f(\cdot)$ satisfy (2.1) and let (2.4), and let $u_{\theta}$ be the solution of the following elliptic equation:

$$
\left\{\begin{array}{l}
-\Delta u+f(u)+\theta u=g(x) \in H^{-1} \quad \text { in } \Omega \\
\left.u\right|_{\partial \Omega}=0
\end{array}\right.
$$

where $\theta>l$. Then

$$
\left\|\nabla u_{\theta}\right\| \rightarrow 0 \quad \text { as } \theta \rightarrow \infty .
$$

Then, as in 30, combining with Lemma 6.1 we can take $\eta_{0}$ (in (2.5) ) large enough such that (recall that $\phi(x)$ is the unique solution of (2.5))

$$
\frac{1}{2}\|\nabla \varphi\|^{2}+2\langle h(\varphi+\phi)-h(\phi), v\rangle-\left\langle h^{\prime}(\phi) \varphi, \varphi\right\rangle \geq 0 \quad \text { for any } \varphi \in H_{0}^{1}(\Omega),
$$

and define

$$
h(s)=f(s)+\eta_{0} s \quad \text { for all } s \in \mathbb{R} .
$$


6.1. Decomposition of the equation. We first decompose the solution $S(t)\left(u_{0}, v_{0}\right)=\left(u(t), u_{t}(t)\right)$ into the sum

$$
S(t) \xi_{u}(0)=K(t) \xi_{u}(0)+D(t) \xi_{u}(0),
$$

where $K(t) \xi_{u}(0)=\left(w(t), w_{t}(t)\right)$ and $D(t) \xi_{u}(0)=\left(z(t), z_{t}(t)\right)$ solve the following equations, respectively:

$$
\left\{\begin{array}{l}
w_{t t}-\Delta w_{t}-\Delta w-\Delta w_{t t}+f(u)-f(z)=\eta_{0} z \quad \text { in } \Omega \times \mathbb{R}^{+} \\
\left.w\right|_{\partial \Omega}=0, \quad\left(w(x, 0), w_{t}(x, 0)\right)=(0,0)
\end{array}\right.
$$

and

$$
\left\{\begin{array}{l}
z_{t t}-\Delta z_{t}-\Delta z-\Delta z_{t t}+h(z)=g(x) \quad \text { in } \Omega \times \mathbb{R}^{+} \\
\left.z\right|_{\partial \Omega}=0, \quad\left(z(x, 0), z_{t}(x, 0)\right)=\xi_{u}(0)
\end{array}\right.
$$

Then, we further decompose the solution $z(x, t)$ of (6.4) as $z(x, t)=v(x, t)+\phi(x)$, where $\phi(x)$ is the unique solution of (2.5) and $v(x, t)$ solves the following equation:

$$
\left\{\begin{array}{l}
v_{t t}-\Delta v_{t}-\Delta v-\Delta v_{t t}+h(z)-h(\phi)=0 \quad \text { in } \Omega \times \mathbb{R}^{+} \\
\left.v\right|_{\partial \Omega}=0, \quad\left(v(x, 0), v_{t}(x, 0)\right)=\xi_{u}(0)-(\phi(x), 0)
\end{array}\right.
$$

6.2. A priori estimates. At first, for the solution of (6.4), from Remark 4.2, we have the following estimate:

Lemma 6.2. There exists $Q_{3}(\cdot) \in \mathfrak{J}$ such that for any bounded set $B \subset \mathcal{H}$, the following estimate holds: for any $t \geq 0$,

$$
\|\nabla z(t)\|^{2}+\int_{0}^{t}\left\|\nabla z_{t}(s)\right\|^{2} d s \leq Q_{3}\left(\|B\|_{\mathcal{H}}+\|g\|_{H^{-1}}\right), \quad \forall\left(z(x, 0), z_{t}(x, 0)\right) \in B .
$$

Second, for the solution of (6.5) we have the following results:

Lemma 6.3. There exist a positive constant $k_{1}$ and $Q_{4}(\cdot) \in \mathfrak{J}$ such that for any bounded set $B \subset \mathcal{H}$, the following estimate holds:

$$
\left\|\left(v(x, t), v_{t}(x, t)\right)\right\|_{\mathcal{H}} \leq Q_{4}\left(\|B\|_{\mathcal{H}}\right) e^{-k_{1} t}, \quad \forall t \geq 0,\left(v(x, 0), v_{t}(x, 0)\right) \in B .
$$

Consequently, for the solution of (6.4) the following estimate holds:

$$
\left\|\left(z(x, t), z_{t}(x, t)\right)-(\phi(x), 0)\right\|_{\mathcal{H}} \leq Q_{4}\left(\|B\|_{\mathcal{H}}\right) e^{-k_{1} t}, \quad \forall t \geq 0, \xi_{0} \in B .
$$

Proof. Multiplying (6.5) by $v_{t}+\epsilon v$, we have

$$
\frac{d}{d t} E_{5 v}(t)+\epsilon E_{5 v}(t)+G_{5 v}(t)+\frac{\epsilon}{2}\|\nabla v(t)\|^{2}=2\left\langle\left(h^{\prime}(z)-h^{\prime}(\phi)\right) z_{t}, v\right\rangle,
$$

where

$$
\begin{aligned}
E_{5 v}(t)=\left\|v_{t}(t)\right\|^{2}+ & (1+\epsilon)\|\nabla v(t)\|^{2}+\left\|\nabla v_{t}(t)\right\|^{2}+2 \epsilon\left\langle v_{t}(t), v(t)\right\rangle \\
& +2 \epsilon\left\langle\nabla v_{t}(t), \nabla v(t)\right\rangle+2\langle h(z)-h(\phi), v(t)\rangle-\left\langle h^{\prime}(\phi) v, v\right\rangle
\end{aligned}
$$

and

$$
\begin{gathered}
G_{5 v}(t)=2\left\|\nabla v_{t}(t)\right\|^{2} \\
+\frac{\epsilon}{2}\|\nabla v(t)\|^{2}-3 \epsilon\left\|v_{t}\right\|^{2}-3 \epsilon\left\|\nabla v_{t}\right\|^{2}-2 \epsilon^{2}\left\langle v_{t}, v\right\rangle \\
-2 \epsilon^{2}\left\langle\nabla v_{t}, \nabla v\right\rangle-\epsilon^{2}\|\nabla v\|^{2}+\epsilon\left\langle h^{\prime}(\phi) v, v\right\rangle .
\end{gathered}
$$

Noticing (6.1), by further taking $\epsilon$ small enough, we have

$$
E_{5 v}(t) \geq \frac{1}{4}\left\|\left(v(t), v_{t}(t)\right)\right\|_{\mathcal{H}}^{2} \quad \text { for all } t \geq 0
$$


and

$$
G_{5 v}(t) \geq 0 \text { for all } t \geq 0 \text {. }
$$

Moreover, from (2.3) and using the Hölder inequality, we have

$$
\begin{aligned}
2\left\langle\left(h^{\prime}(z)-h^{\prime}(\phi)\right) z_{t}, v\right\rangle & =2\left\langle h^{\prime \prime}(r z+(1-r) \phi) z_{t}, v^{2}\right\rangle \\
& \leq \begin{cases}C \int_{\Omega}\left(1+|z|^{\frac{6-N}{N-2}}+|\phi|^{\frac{6-N}{N-2}}\right)\left|z_{t} \| v\right|^{2} d x & N=3,4,5, \\
C \int_{\Omega}\left|z_{t} \| v\right|^{2} d x & N \geq 6\end{cases} \\
& \leq c_{3}\left\|\nabla z_{t}\right\|\|\nabla v\|^{2} \leq \frac{\epsilon}{2}\|\nabla v\|^{2}+\frac{c_{3}}{\epsilon}\left\|\nabla z_{t}\right\|^{2} E_{5 v}(t),
\end{aligned}
$$

where $r \in(0,1)$ and the constant $c_{3}$ depends only on $\|B\|_{\mathcal{H}}+\|\nabla \phi\|$.

Therefore, from Lemma 6.2 and noticing $E_{5 v}(0) \leq Q\left(\|B\|_{\mathcal{H}}+\|\nabla \phi\|\right)$, applying Lemma 3.3 we can complete our proof immediately.

For the solution of (6.3), the same as Lemma 5.3 , for each $\alpha \in\left[0, \min \left\{1, \frac{N}{2}-1\right\}\right)$, by multiplying (6.3) by $A^{\alpha} w_{t}$, we have

Lemma 6.4. For any $\alpha \in\left[0, \min \left\{1, \frac{N}{2}-1\right\}\right)$, there exist $k_{\alpha}>0$ and $Q_{\alpha}(\cdot) \in \mathfrak{J}$ such that for any $t \geq 0$,

$$
\left\|K(t) \xi_{u}(0)\right\|_{\mathcal{H}^{\alpha}}^{2} \leq Q_{\alpha}\left(\left\|\xi_{u}(0)\right\|_{\mathcal{H}}\right) e^{k_{\alpha} t},
$$

where $k_{\alpha}$ also depends on $\left\|\xi_{u}(0)\right\|_{\mathcal{H}}$.

Now, similar to Lemma 5.4 based on Lemma 6.2 and Lemma 6.3 we can decompose $u(t)$ as follows:

Lemma 6.5. Let $\left(u(t), u_{t}(t)\right)$ be the solution of $\left(E_{1}\right)$ corresponding to the initial data $\xi_{u}(0)=\left(u_{0}, v_{0}\right)$. Then, for any $\eta>0$, we can decompose $u(t)$ as

$$
u(t)=v_{1}(t)+w_{1}(t), \quad \text { for all } t \geq 0,
$$

where $v_{1}(t)$ and $w_{1}(t)$ satisfy the following estimates:

$$
\int_{s}^{t}\left\|\nabla v_{1}(\tau)\right\|^{2} d \tau \leq \eta(t-s)+C_{\eta} \text { for all } t \geq s \geq 0
$$

and

$$
\left\|A^{\frac{1+\alpha}{2}} w_{1}(t)\right\|^{2} \leq K_{\eta} \text { for all } t \geq 0,
$$

with the constants $C_{\eta}$ and $K_{\eta}$ depending on $\eta,\left\|\xi_{u}(0)\right\|_{\mathcal{H}}$ and $\|g\|_{H^{-1}}$.

Moreover, $v_{1}(\cdot, t)$ and $w_{1}(\cdot, t)$ satisfy the following estimates respectively:

$$
\left\|\nabla v_{1}(t)\right\| \leq Q_{5}\left(\|B\|_{\mathcal{H}}\right) \quad \text { for all } t \geq 0
$$

and

$$
\left\|\nabla w_{1}(t)\right\|=\left\|\nabla\left(u(t)-v_{1}(t)\right)\right\| \leq Q_{6}\left(\|B\|_{\mathcal{H}}\right) \quad \text { for all } t \geq 0 .
$$

Proof. The proof is the same as that in [30, Lemma 4.5]. 
6.3. Proof of Theorem 2.2, We will follow the idea from [37, and the details similar to [28.

Proof of Theorem 2.2. We decompose our proof into two steps for clarity.

Step 1. We first claim that

For each $\alpha \in\left[0, \min \left\{1, \frac{N}{2}-1\right\}\right)$, there exists a constant $J_{B, \alpha}$, which depends only on the $\mathcal{H}$-bounds of $B(\subset \mathcal{H})$ and $\alpha$, such that

$$
\left\|K(t) \xi_{u}(0)\right\|_{\mathcal{H}^{\alpha}}^{2}=\left\|\left(w(t), w_{t}(t)\right)\right\|_{\mathcal{H}^{\alpha}}^{2} \leq J_{B, \alpha} \quad \text { for all } t \geq 0 \text { and } \xi_{u}(0) \in B .
$$

Multiplying (6.3) by $A^{\alpha}\left(w_{t}(t)+\epsilon w(t)\right)$, we obtain that

$$
\begin{aligned}
\frac{1}{2} \frac{d}{d t}( & \left.\left\|A^{\frac{\alpha}{2}}\left(w_{t}+\epsilon w\right)\right\|^{2}+\mu\left\|A^{\frac{1+\alpha}{2}}\left(w_{t}+\epsilon w\right)\right\|^{2}\right)-\left\langle\epsilon w_{t}, A^{\alpha}\left(w_{t}+\epsilon w\right)\right\rangle \\
& -\left\langle\epsilon w_{t}, A^{1+\alpha}\left(w_{t}+\epsilon w\right)\right\rangle-\left\langle\Delta w_{t}, A^{\alpha}\left(w_{t}+\epsilon w\right)\right\rangle-\left\langle\Delta w, A^{\alpha}\left(w_{t}+\epsilon w\right)\right\rangle \\
=- & \left.f(u)-f(z), A^{\alpha}\left(w_{t}+\epsilon w\right)\right\rangle+\left\langle\eta_{0} z, A^{\alpha}\left(w_{t}+\epsilon w\right)\right\rangle,
\end{aligned}
$$

where $\epsilon(>0)$ is small enough and will be determined later.

Then, as in [28, Lemma 4.4], we can obtain the claim above by applying Lemma 6.5 to overcome the difficulty from the critical nonlinearity.

Step 2. Applying Lemma 6.3 and Step 1 to $B_{0}$ (recall $B_{0} \subset \mathcal{H}$ is the bounded absorbing set given in $\S 3$ ), also using the attraction transitivity lemma devised in [15], we can finish our proof by setting: for each $\alpha \in\left[0, \min \left\{1, \frac{N}{2}-1\right\}\right)$,

$$
\mathcal{B}_{\alpha}=\left\{z \in \mathcal{H}:\|z-(\phi(x), 0)\|_{\mathcal{H}^{\alpha}}^{2} \leq J_{B_{0}, \alpha}\right\},
$$

where $J_{B_{0}, \alpha}$ is the constant given in (6.15) corresponding to $B_{0}$.

In the following we state a decomposition result about $u(t)$, which can be used to construct a finite dimensional exponential attractor (e.g., see [14, 23, 30]); its proof is the same as that in [30, Lemma 4.9].

Lemma 6.6. Under the assumption of Theorem 2.1, for any bounded (in $\mathcal{H}^{\alpha}$, $\alpha \in\left[0, \min \left\{\frac{N}{2}-1\right\}\right)$ ) subset $B_{1} \subset \mathcal{H}^{\alpha}$, if the initial data $\xi_{u}(0) \in \phi(x)+B_{1}$, then the solution $u(t)$ of $\left(E_{1}\right)$ also satisfies a similar estimate; more precisely, we have

$$
\left\|S(t) \xi_{u}(0)-(\phi(x), 0)\right\|_{\mathcal{H}^{\alpha}}^{2}=\left\|\left(u(t), u_{t}(t)\right)-(\phi(x), 0)\right\|_{\mathcal{H}^{\alpha}}^{2} \leq K_{B_{1}}
$$

for any $t \geq 0$ and any $\xi_{u}(0) \in \phi(x)+B_{1}$; where the constant $K_{B_{1}}$ depends only on $\alpha$ and the $\mathcal{H}^{\alpha}$-bound of $B_{1}$.

Remark 6.7. Based on Theorem 2.2 and Lemma 6.6, we can construct a finite dimensional exponential attractor $\mathcal{E}$ for $\{S(t)\}_{t \geq 0}$ under Assumption II. Moreover, we can decompose $\mathcal{E}$ as $\mathcal{E}=(\phi(x), 0)+\mathcal{E}^{\prime}$, where $\mathcal{E}^{\prime}$ is bounded in $\mathcal{H}^{\alpha}$ for any $\alpha \in\left[0, \min \left\{1, \frac{N}{2}-1\right\}\right)$.

\section{ACKNowledgments}

The authors are grateful to the anonymous referee for the comments improving the original version of the manuscript. This paper was prepared while the first author was visiting The University of Chicago. He wishes to express his thanks for the hospitality of the Department of Mathematics, The University of Chicago. 


\section{REFERENCES}

1. J. Arrieta, A.N. Carvalho and J.K. Hale, A damped hyperbolic equation with critical exponent, Comm. Partial Differential Equations 17 (1992), 841-866. MR.1177295 (93f:35145)

2. A.V. Babin and M.I. Vishik, Attractors of evolution equations, North-Holland, Amsterdam, 1992. MR 1156492 (93d:58090)

3. I.L. Bogolubsky, Some examples of inelastic soliton interaction, Comput. Phys. Comm. 13 (1977), 149-155.

4. A.N. Carvalho and J.W. Cholewa, Local well posedness for strongly damped wave equations with critical nonlinearities, Bull. Austral. Math. Soc. 66 (2002), 443-463. MR1939206 (2004b:35228)

5. A.N. Carvalho and J.W. Cholewa, Attractors for strongly damped wave equations with critical nonlinearities, Pacific J. Math. 207 (2002), 287-310. MR.1972247 (2004b:35023)

6. A.N. Carvalho and J.W. Cholewa, Local well posedness, asymptotic behavior and asymptotic bootstrapping for a class of semilinear evolution equations of the second order in time, Trans. Amer. Math. Soc. 361 (2009), 2567-2586. MR2471929

7. A.N. Carvalho, J.W. Cholewa and T. Dlotko, Strongly damped wave problems: bootstrapping and regularity of solutions, J. Differential Equations 244 (2008), 2310-2333. MR2413843 (2009c:35303)

8. Y. Cho and T. Ozawa, On small amplitude solutions to the generalized Boussinesq equations, Discrete Contin. Dyn. Syst., 17 (2007), 691-711. MR2276469 (2008c:35268)

9. J.W. Cholewa, R. Czaja and G. Mola, Remarks on the fractal dimension of bi-space global and exponential attractors, Boll. Un. Math. Ital. 1 (2008), 121-145. MR.2388001(2009i:37199)

10. P.A. Clarkson, R.J. Leveque and R.A. Saxton, Solitary wave interaction in elastic rods, Stud. Appl. Math. 75 (1986), 95-122. MR859173 (87j:73034)

11. F. Di Plinio and V. Pata, Robust exponential attractors for the strongly damped wave equation with memory. I, Russian J. Math. Physics 15 (2008), 301-315. MR.2448344 (2010c:35136)

12. F. Di Plinio, V. Pata and S.V. Zelik, On the strongly damped wave equation with memory, Indiana Univ. Math. J. 57 (2008), 757-780. MR2414334 (2009m:35342)

13. M. Conti and V. Pata, On the regularity of global attractors, Discrete Contin. Dyn. Syst. 25 (2009), 1209-1217. MR2552136

14. M. Efendiev, A. Miranville and S.V. Zelik, Exponential attractors and finite-dimensional reduction of non-autonomous dynamical systems, Proc. Royal Soc. Edin. 135A (2005), 703730. MR2173336 (2007a:37098)

15. P. Fabrie, C. Galusinski, A. Miranville and S.V. Zelik, Uniform exponential attractors for a singular perturbed damped wave equation, Discrete Contin. Dyn. Syst. 10 (2004), 211-238. MR2026192 (2006c:37088)

16. J.M. Ghidaglia and A. Marzocchi, Longtime behavior of strongly damped wave equations, global attractors and their dimension, SIAM J. Math. Anal. 22 (1991), 879-895. MR1112054 (92e:35033)

17. M. Grasselli and V. Pata, Asymptotic behavior of a parabolic-hyperbolic system, Comm. Pure Appl. Anal. 3 (2004), 849-881. MR2106302 (2005h:35150)

18. J.K. Hale, Asymptotic Behavior of Dissipative Systems, Amer. Math. Soc., Providence, RI, 1988. MR941371 (89g:58059)

19. T. Kano and T. Nishida, A mathematical justification for Korteweg-de Vries equation and Boussinesq equation of water surface waves, Osaka J. Math. 23 (1986), 389-413. MR856894 $(87 \mathrm{j}: 35325)$

20. V.G. Makhankov, On stationary solutions of the Schrödinger equation with a self-consistent potential satisfying Boussinesq's equation, Phys. Lett. A 50 (1974), 42-44.

21. V.G. Makhankov, Dynamics of classical solitons (in non-integrable systems), Physics reports, Phys. Lett. C 35 (1978), 1-128. MR481361 (80i:81044)

22. A. Miranville and S.V. Zelik, Attractors for Dissipative Partial Differential Equations in Bounded and Unbounded Domains. Handbook of differential equations, Evolutionary Equations, Volume 4, C.M. Dafermos and M. Pokorny, eds., Elsevier, Amsterdam, 2008, p.103. MR2508165 (2010c:37175)

23. V. Pata and M. Squassina, On the strongly damped wave equation, Comm. Math. Phys. 253 (2005), 511-533. MR2116726 (2005k:35291) 
24. V. Pata and S.V. Zelik, A remark on the damped wave equation, Commun. Pure Appl. Anal. 5 (2006), 611-616. MR 2217604 (2006m:35254)

25. V. Pata and S.V. Zelik, Smooth attractors for strongly damped wave equations, Nonlinearity 19 (2006), 1495-1506. MR2229785 (2007f:35257)

26. G. Raugel, Global attractors in partial differential equations, in: B. Fiedler (Ed.), Handbook of Dynamical Systems, Vol. 2, Elsevier, Amsterdam, 2002, pp. 885-982. MR.1901068 (2003f:37151)

27. Y. Shang, Initial-boundary value problem for the equation $u_{t t}-\Delta-\Delta u_{t}-\Delta u_{t t}=f(u)$, Acta Math. Appl. Sinica (Chinese Ser.) 23 (2000), 385-393. MR.1797635 (2001k:35221)

28. C. Sun, D. Cao and J. Duan, Non-autonomous wave dynamics with memory - Asymptotic regularity and uniform attractor, Discrete Contin. Dyn. Syst., Series B 9 (2008), 743-761. MR 2379435 (2009g:35204)

29. C. Sun and M. Yang, Dynamics of the nonclassical diffusion equations, Asymptotic Analysis 59 (2008), 51-81. MR2435673 (2009m:35056)

30. C. Sun, Asymptotic regularity for some dissipative equations, J. Differential Equations 248 (2010), 342-362. MR2558170

31. S. Wang and G. Chen, The Cauchy problem for the generalized IMBq equation in $W^{s, p}\left(\mathbb{R}^{n}\right)$, J. Math. Anal. Appl. 266 (2002), 38-54. MR1876769 (2002k:35222)

32. G.F. Webb, Existence and asymptotic behavior for a strongly damped nonlinear wave equation, Canad. J. Math. 32 (1980), 631-643. MR586981 (81i:35116)

33. Y. Xie and C. Zhong, The existence of global attractors for a class nonlinear evolution equation, J. Math. Anal. Appl. 336 (2007), 54-69. MR2348490(2009b:35303)

34. Y. Xie and C. Zhong, Asymptotic behavior of a class of nonlinear evolution equations, Nonlinear Anal. TMA 71 (2009), 5095-5105. MR2560179

35. M. Yang and C. Sun, Dynamics of strongly damped wave equations in locally uniform spaces: attractors and asymptotic regularity, Trans. Amer. Math. Soc. 361 (2009), 1069-1101. MR:2452835 (2010b:37219)

36. M. Yang and C. Sun, Exponential attractors for the strongly damped wave equations, Nonlinar Anal., Real World Appl. 11 (2010), 913-919. MR2571264

37. S.V. Zelik, Asymptotic regularity of solutions of a nonautonomous damped wave equation with a critical growth exponent, Commun. Pure Appl. Anal. 3 (2004), 921-934. MR.2106304 (2005h:35249)

38. H. Zhang and Q. Hu, Existence and stability of the global weak solution of a nonlinear evolution equation, Acta Math. Sci. Ser. A Chin. Ed. 24 (2004), 329-336. MR2072406(2005d:35180)

School of Mathematics and Statistics, Lanzhou University, Lanzhou, 730000, PeoPLE's Republic OF CHINA

E-mail address: sunchunyou@gmail.com; sunchy@lzu.edu.cn

School of Mathematics and Statistics, Lanzhou University, Lanzhou, 730000, PeoPLE's Republic of China

E-mail address: yanglu@lzu.edu.cn

Department of Applied Mathematics, Illinois Institute of Technology, Chicago, IlliNOIS 60616

E-mail address: duan@iit.edu 